$\frac{12}{8-4-95} 850$

UCRL-ID-118664

The Effect of Actinide Thin Films on the Electronic Structure and Reactivity of Various Elements

T. Gouder

C. Colmenares

December 8, 1994

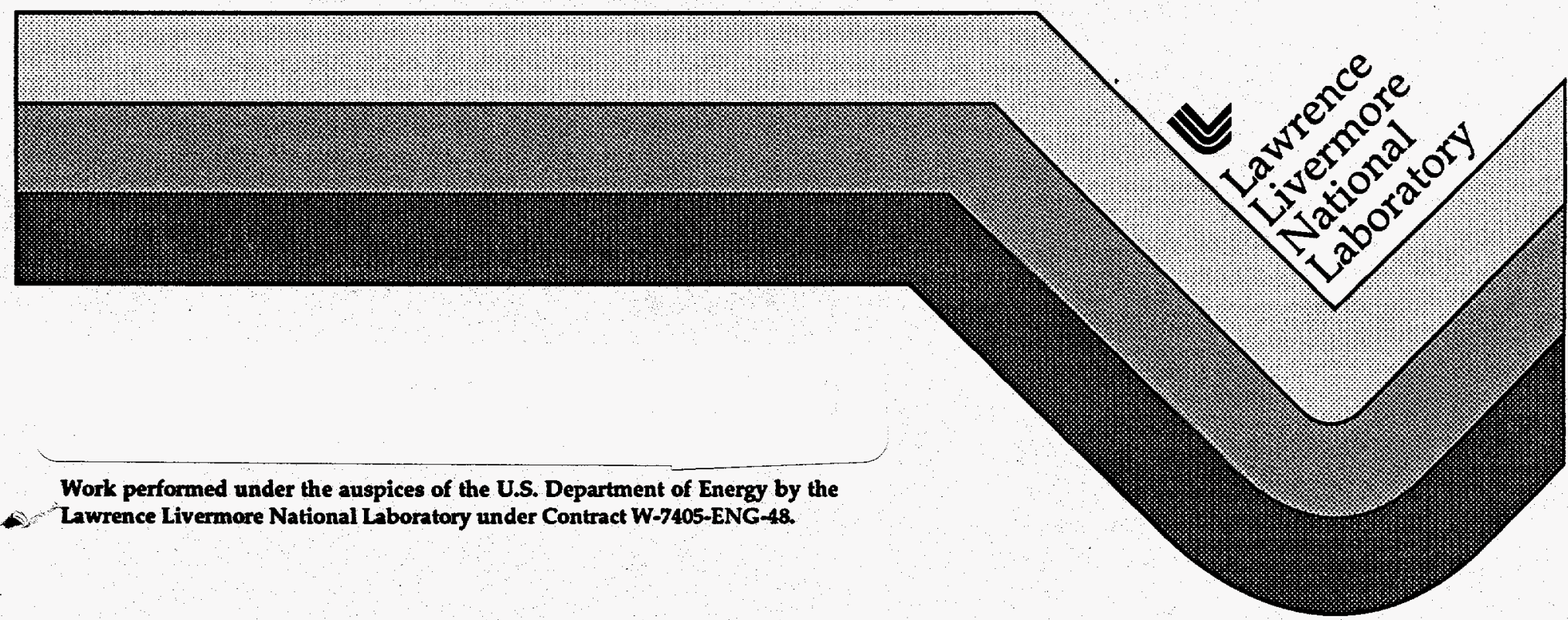




\section{DISCLAIMER}

This document was prepared as an account of work sponsored by an agency of the United States Government. Neither the United States Covernment nor the University of California nor any of their employees, makes any warranty, express or implied, or assumes any legal liability or responsibility for the accuracy, completeness, or usefulness of any information, apparatus, product, or process disclosed, or represents that its use would not infringe privately owned rights. Reference herein to any specific commercial product, process, or service by trade name, trademark, manufacturer, or otherwise, does not necessarily constitute or imply its endorsement, recommendation, or favoring by the United States Government or the University of California. The views and opinions of authors expressed herein do not necessarily state or reflect those of the United States Covernment or the University of California, and shall not be used for advertising or product endorsement purposes.

This report has been reproduced directly from the best available copy.

Available to DOE and DOE contractors from the

Office of Scientific and Technical Information

P.O. Box 62, Oak Ridge, IN 37831

Prices available from (615) 576-8401, FTS 626-8401

Available to the public from the

National Technical Information Service

U.S. Department of Commerce

5285 Port Royal Rd.,

Springfield, VA 22161 


\section{DISCLAIMER}

Portions of this document may be illegible in electronic image products. Images are produced from the best available original document. 


\title{
The Effect of Actinide Thin Films on the Electronic Structure and Reactivity of Various Elements
}

\author{
T.H. Gouder \\ C.A. Colmenares
}

Lawrence Livermore National Laboratory

December 8, 1994 
The Effect of Actinide Thin Films

on the Electronic Structure and Reactivity of Various Elements

T.H. Gouder

C.A. Colmenares

\section{Table of Contents}

Page

Abstract _ i

1. Introduction 1

2. Overlayer Growth, Interdiffusion, and Electronic Structure 4

3. Surface Reactions on Thin U Overlayers 14

a. Corrosion-Induced Interface Decomposition 14

b. Use of Thin Layers to Study Terminal/Oxidation Reaction Products 18

c. U-Doped Surfaces: Actinides as Promoters in Catalysis 21

4. Bulk Diffusion of U Overlayers 27

$\begin{array}{ll}\text { 5. Conclusions } & 36\end{array}$

$\begin{array}{ll}\text { 6. Author's Comments } & 37\end{array}$

$\begin{array}{lll}\text { 7. } & \text { References } & 38\end{array}$ 


\begin{abstract}
This report summarizes the experimental work carried out at the Lawrence Livermore National Laboratory on the electronic structure and reactivity of uranium thin films on $\mathrm{Pd}, \mathrm{Pt}$, Si, graphite, $\mathrm{Cu}$, and $\mathrm{Au}$ substrates from 1990 to 1993. The U-Pd system was studied in the most detail because it was the first to be chosen right after the completion of the experimental equipment.

We first studied and characterized clean $U$ overlayers and the possible surface reactions between this metal and the substrates studied. We then subjected these systems to reactive conditions such as heating and adsorbing corrosive gases $\left(\mathrm{O}_{2}, \mathrm{CO}, \mathrm{CO}_{2}\right.$, and $\left.\mathrm{C}_{2} \mathrm{H}_{4}\right)$. Finally we investigated the diffusion of $\mathrm{U}$ metal and some of its compounds into the substrates.

A new technique was developed, based on Auger Electron Spectroscopy, to follow in real time the diffusion of $U$ overlayers into the substrate. The temperature of the sample is ramped linearly up to $900^{\circ} \mathrm{C}$ while following the Auger peak intensities of the two components for a given system. Diffusion rates are obtained by differentiating the measured intensity curves, then peaks result corresponding to diffusion processes with different activation energies. This technique bears a strong similarity to thermal desorption spectroscopy (TDS), where the sample is heated linearly and the rate of desorption is measured as a function of temperature and heating rate.
\end{abstract}




\section{Introduction}

Research on actinides is marked by their ambivalent role in energy production and environmental conservation and, on a scientific level, by their interesting chemical and physical properties. In our research at the Lawrence Livermore National Laboratory (LLNL) we are studying the chemical reactivity and electronic structure of actinide surfaces and interfaces with other metals. We are particularly interested in their interaction with gases (corrosion, catalysis), the decomposition of actinide-transition metal (TM) interfaces, and the diffusion of actinide atoms into a substrate. This research may be of importance in nuclear waste disposal. Modeling long term corrosion and diffusion reactions (100-1000 years) by laboratory experiments needs extrapolation of reaction mechanisms and rates to long times. The reliability of this procedure is determined by our knowledge of the reaction system, which initially is the actinide-containment metal interface. This includes the more fundamental aspect of the electronic structure of these systems, which will ultimately determine their chemical properties.

We have used thin layers of actinides (uranium at this stage) to simulate the actinide-containment metal interface. Thin layers are accessible to surface analysis tools and can be studied for their resistance to corrosion and heat (Figure 1). In addition we can study the bulk diffusion behavior of actinide atoms by measuring their surface concentration changes as a function of time and temperature, which gives information on the kinetics of diffusion.

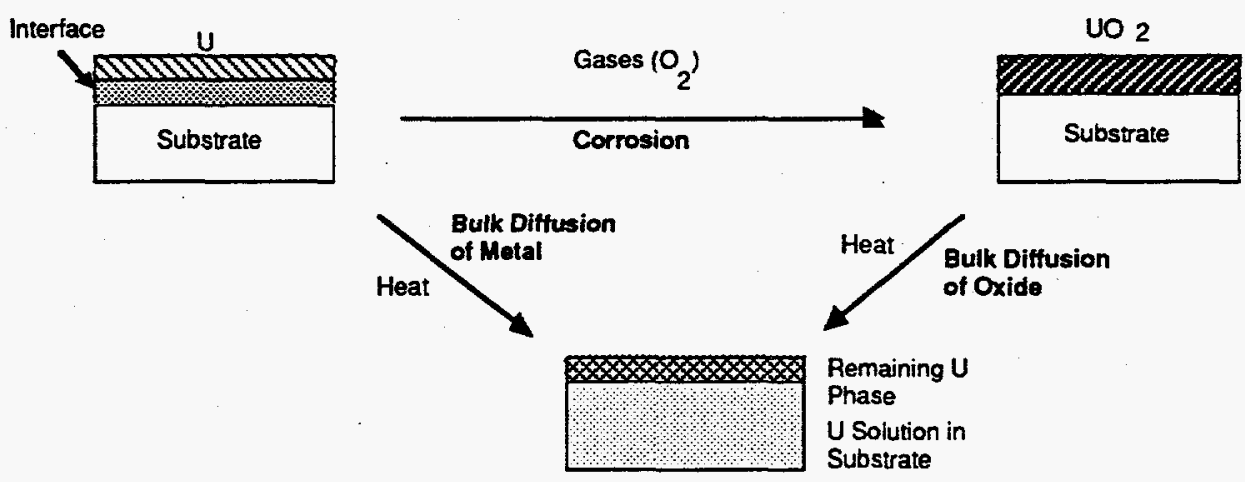

Fig. 1 Thin layers can undergo various chemical processes such as corrosion and heat induced interface decomposition. These reactions may also cause problems during handling and long term storage of nuclear waste.

On a more fundamental level, thin layers allow the accentuation of some of the most interesting physical and chemical properties of actinides. The decreased coordination of actinide atoms on a surface becomes even more pronounced in thin layers and clusters, and can be expected to result in further narrowing of the $5 \mathrm{f}$ bands in early actinides. This may eventually result in the 
breakdown of itinerancy close to the surface, even in those compounds that have itinerant $5 \mathrm{f}$ electrons in the bulk (Figure 2). Mixed valence and enhanced surface magnetism can result in such systems. Finally, actinide deposition onto a surface allows easy and rapid synthesis of surface compounds with variable stoichiometry. Interesting applications lie in the field of catalysis, where we can prepare surfaces doped with minute amounts of $U$ to investigate their adsorptive behavior and ultimately their catalytic properties.

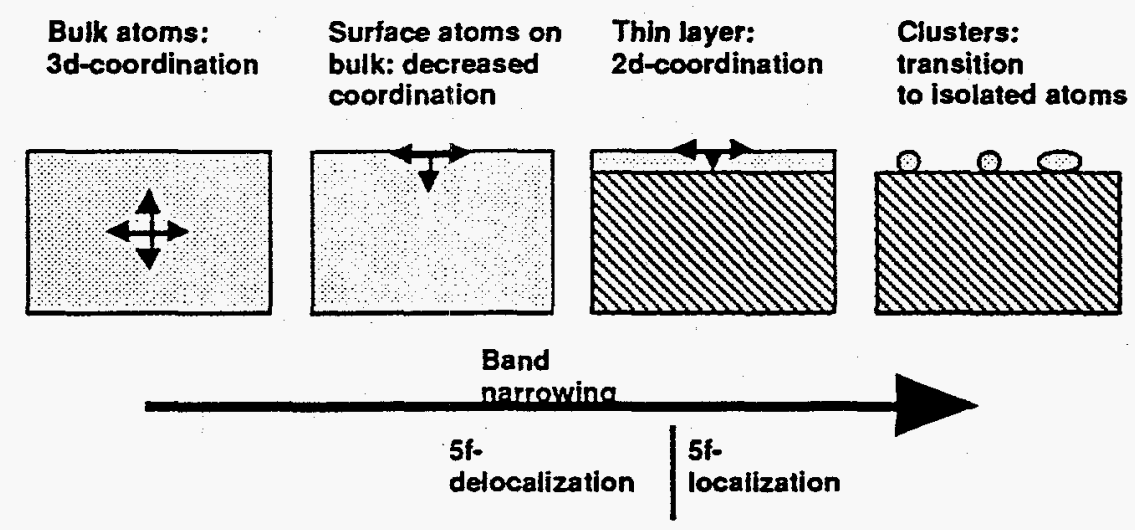

Fig. 2 The coordination of atoms decreases from the bulk to the surface, thin layers and clusters. This may eventually result in the localization of the $5 f$ electrons even in light actinides.

Below we will describe some of the experimental results obtained at this stage of our work. We limited our research to $U$ compounds. Because no previous work has been published on thin layers of $U$, little is known about their stability, spontaneous surface alloying, etc. This determined our experimental strategy (Figure 3). We first studied and characterized clean overlayers and the possible surface reactions between $U$ and the substrate. We then subjected these systems to more reactive conditions (heat, corrosive gases) and finally investigated the bulk diffusion of $U$ metal and compounds into the substrate. We will, therefore, begin with a discussion of the electronic structure of $U$ overlayers on substrates, then proceed to some surface reactions of these systems, and finally address the bulk diffusion behavior of $U$ and $U$ compounds. The substrates were chosen in light of the considerations presented above. We looked for materials that: a) Would favor $5 f$ localization in thin films (Section 2); b) Are potential containment materials (Section 4), and ; c) Are potential catalysts whose properties could be modified by $U$ dopants (Section 3). Thus far we have studied $U$ deposition on Pd, Pt, $\mathrm{Si}$, Graphite, $\mathrm{Cu}$, and $\mathrm{Au}$. The Pd system has been studied in the most detail because it happened that at this point the experimental setup was completed. We studied only polycrystalline substrates because single crystals were not available at the time. Therefore, we will not address the question of epitaxial growth, lattice mismatch, etc. Single-crystal experiments are imminent. 


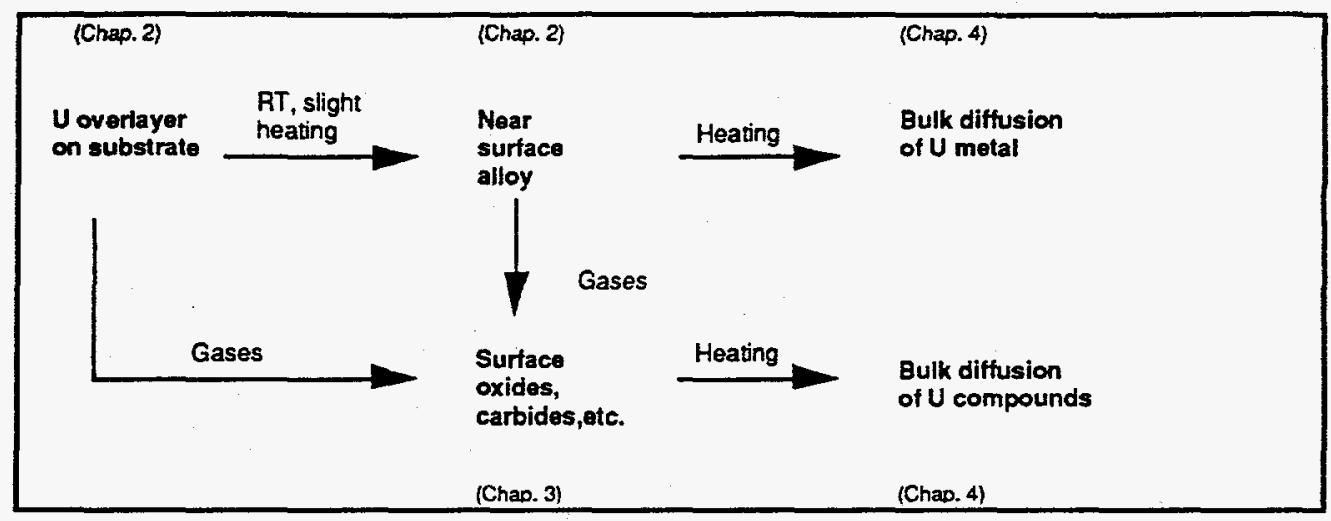

Fig. 3 Organization of $U$ thin layer research at Livermore 


\section{Overlayer Growth, Interdiffusion, and Electronic Structure}

We will first discuss how $U$ deposits on the substrates and whether it forms an overlayer or spontaneously reacts with the substrate. Because the mode of growth is reflected by the electronic structure of the surface we will discuss both issues together. As shown in Figure 2 we can expect that the decreased coordination at the surface and in thin layers results in a narrowing of the $5 \mathrm{f}$ bands. This phenomenon is also experienced by transition metal surface atoms ${ }^{1,2}$. Because the $5 \mathrm{f}$ bandwidth is already narrow in bulk $\mathrm{U}$, additional narrowing at the surface may result in the breakdown of itinerancy, which occurs when the bandwidth gets smaller than the f-f correlation energy. But it is not clear at all if this will easily happen for $U$, because the $5 f$ bandwidth might still be too large; in Pu the changes for observing localization are much better! Let us discuss the chances for inducing $5 f$ localization in $U$. We know that in $U$ metal and most of its alloys $U$ surface atoms have delocalized $5 f$ electrons due to their bonding interaction with the underlying bulk, either by direct $5 \mathrm{f}-5 \mathrm{f}$ overlap or by $5 \mathrm{f}$ hybridization with other orbitals. This has been shown by highly surface-sensitive studies of the electronic structure of $U$ bulk compounds (UPS studies). But while such interactions are also expected for thin layers of $U$ on various substrates, these systems differ favorably from bulk alloys in several ways: a) The $U$ surface concentration (or coverage) can be varied continuously and a highly diluted $U$ surface phase may be synthesized. By doing this we suppress the direct $U-U$ interactions that contribute considerably to U5f delocalization in alloys. b) We can choose substrates that only interact weakly with $U$, and on the extreme, we find graphite on which $U$ deposits as clusters ${ }^{3}$, while for bulk compounds there has to be a minimal interaction to avoid phase separation. This interaction might be responsible for $5 \mathrm{f}$ delocalization in most metallic bulk compounds. However, not only the strength of the overall interaction, but rather the involvement of the $5 \mathrm{f}$-electrons in bonding determines their delocalization. This latter aspect has influenced our choice of substrates. We started with a polycrystalline Pd substrate because UPd 3 is the only known U intermetallic where the 5 f-electrons are localized. This happens because they are not hybridized with the Pd4d states, and because the $U$ atoms are spaced far enough apart (due to the specific crystalline structure of $\mathrm{UPd}_{3}$ ) to minimize direct $5 \mathrm{f}-5 \mathrm{f}$ overlap. Therefore, we would expect that interactions between a $U$ overlayer and a Pd substrate would not induce $5 f$ delocalization. We then proceeded to $\mathrm{Pt}$, which forms the heavy-fermion $\mathrm{UP}_{3}$, whose $5 \mathrm{f}$ electrons are found in very narrow bands. Again we hoped that the interaction between surface $U$ and the $P t$ substrate would contribute little to $5 \mathrm{f}$ delocalization. This expectation may have been a little naive because it has been argued that the surface leads to the breakdown of heavy-fermion properties and, in addition, $5 \mathrm{f}$ localization was not observed for surface atoms of bulk heavy fermions. 
We produced thin layers of $U$ at room temperature by magnetron sputter deposition. Coverages from the submonolayer range to micron thickness were achieved by this technique ${ }^{4}$. The coverage was determined by the ratio of the Uovv/PdMNN Auger intensities. However, the interpretation of these data is not unambiguous because it depends on the way $U$ deposits on the surface. If $U$ spontaneously diffuses into the near surface region to form a near surface alloy the dosage should be described in terms of $U$ concentration. If $U$ stays on the top surface forming an overlayer, $U$ dosage should be described by partial coverage and overlayer thickness. We found that actually both interdiffusion and overlayer/island formation take place simultaneously (see below). Therefore, we will describe the $U$ dosage (for $\mathrm{Pd}$ ) in terms of the UOVv/PdMNN AES intensity ratio. To give the reader a guideline, the ratios correspond approximately to the atomic ratios if $\mathrm{U}$ diffuses into Pd to form a uniform near-surface alloy within the AES information depth, which is about four monolayers. For the other substrates, the dosage was determined by the ratio of the main XPS emissions, e.g., U4f/Pt4f, U4f/Si3d, U4f/C1s, and is also interpreted in terms of $U$ concentration in a uniform near-surface alloy.

Figure 4 shows the U4f spectra for $U$ deposited on Pd. At very low dosage (0.23) the U4f emission is narrow, symmetric, and has a low inelastic background. This shows that $U$ deposits on the top surface (low inelastic background) to form a thin overlayer and does not agglomerate in clusters (sharp $U 4 f$ signal suggesting $U$ in one well-defined environment). The fairly symmetric shape suggests a low local density of states of the $U$ atoms (DOS) at the Fermi-level $\left(E_{F}\right)$. (According to the Doniach-Sunjic theory ${ }^{5}$ electrons at the Fermi-level inelastically scatter the photoelectrons by creation of electron-hole pairs; a high DOS at $\mathrm{E}_{\mathrm{F}}$ emphasizes this and results in multiple energy losses of the photoelectrons which translates in a loss-tail on the high binding energy side of the photoelectron peak, i.e., the peak becomes asymmetric. For example, this asymmetry has been observed for $\mathrm{Pu}^{6}$, which has a high $\mathrm{DOS}$ at $\mathrm{E}_{\mathrm{F}}$ due to delocalized $5 \mathrm{f}$ states, while it is missing in Am which has a low DOS at $E_{F}$ because the $5 f$ electrons are localized ${ }^{7}$.) Hence the symmetric shape of the U4f emission points to localized U5f electrons, and this is corroborated by UPS data (see below). With increasing $U$ dosage $(U / P d=1.05,1.92)$ the U4f emission broadens, becomes asymmetric and shifts to low BE. Broadening points to the presence of several $\mathrm{U}$ species, either a $\mathrm{U}$ overlayer covered by islands or $\mathrm{U}$ diffusing into $\mathrm{Pd}$.

Later we will present evidence for island formation at high dosage (Section 4), but there is also strong evidence for inward diffusion of $U$ : the Pd3d levels experience a chemical shift to higher $\mathrm{BE}$ because of the chemical interaction between $\mathrm{U}$ and $\mathrm{Pd}$, and the Pd spectra corresponding to medium coverage $(\mathrm{U} / \mathrm{Pd}=1.92)$ show most of the $\mathrm{Pd} 3 \mathrm{~d}$ signal shifted. This indicates that most of the Pd atoms within the Pd3d information depth of about 8 monolayers interact with $U$. This 


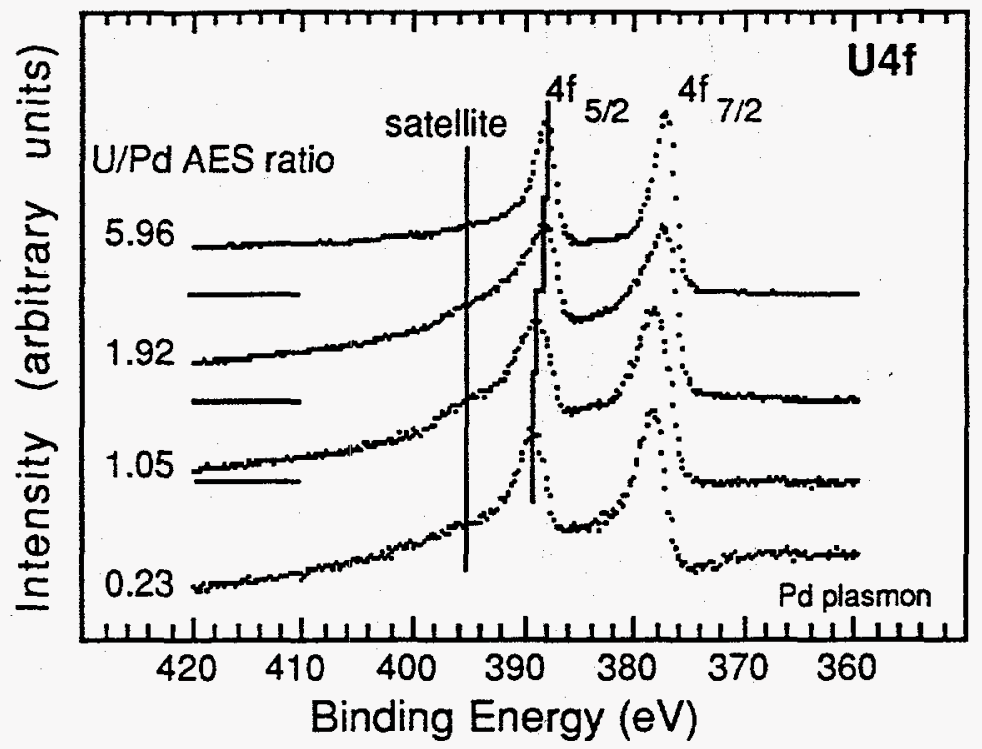

Fig. $4 U$ deposition on $\mathrm{Pd}$ at room temperature. The horizontal lines indicate the baseline at the low BE side and show the inelastic background to increase with $U$ dosage. For very low $U$ dosage $(0.23)$ the low BE baseline is not flat because of a plasmon excitation from the Pd3d emission falling in that region.

can only be explained by $U$ diffusing into the substrate. The increased asymmetry of the $U 4 f$ reflects a high DOS at $\mathrm{E}_{\mathrm{F}}$ which can only be produced by delocalized 5 f electrons; delocalization is induced by the increasing $U$ concentration and is probably due to direct $U-U$ interaction. At very high dosages the U4f line becomes sharper and more symmetric again. It matches the $\mathrm{BE}$ and shape of the $4 \mathrm{f}$ emission of $\mathrm{U}$ metal. The decrease of asymmetry is explained by a decrease of the DOS at $E_{F}$, which is expected to occur when the $5 f$ electrons become more delocalized, i.e., when the band further broadens. The overall picture is shown schematically in Figure 5.

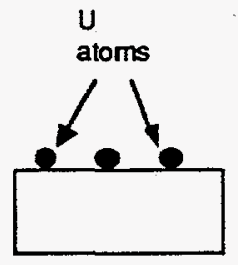

Low $U$ dosage

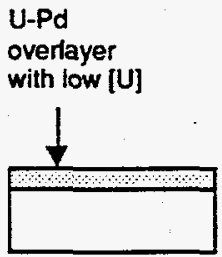

Medium U dosage

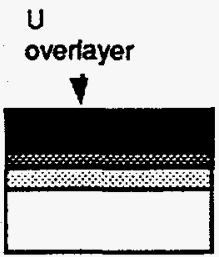

High U dosage

$$
\begin{aligned}
& -U \text { atomically dispersed } \\
& -U \text { at top surface } \\
& \text { - U-Pd interaction } \\
& -5 f \text { localization }
\end{aligned}
$$

$$
\begin{aligned}
& \text { - U-Pd overlayer + islands } \\
& \text { - increasing U concentration } \\
& \text { - U-Pd+U-U interaction } \\
& \text { - beginning } 5 f \text { delocalization }
\end{aligned}
$$

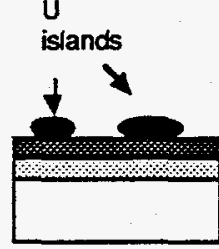

:
- U overlayer

- further increase of $5 \mathrm{f}$ bandwidth

Fig. 5a Mode of growth of $U$ on polycrystalline Pd. A surface phase of dispersed $U$ atoms on $P d$ at low coverage is followed by island growth with partial U-Pd interdiffusion and finally the formation of a thick $U$ film. 


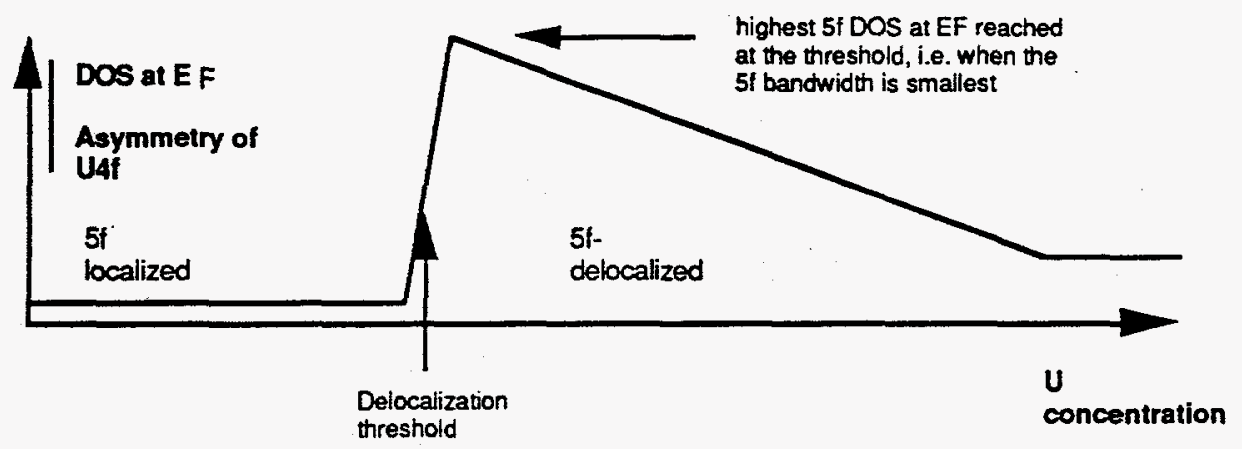

Fig. 5b Evolution of the asymmetry of the $U 4 f$ with $5 f$ bandwidth. The highest asymmetry has to be expected when the $5 f$ electrons are delocalized but sit at the localization threshold, which results in narrow $5 f$ bands with a high $5 f$ DOS at EF.

Let us briefly discuss the evolution of the $6 \mathrm{eV}$ satellite (Figure 4). It is found in UPd 3 where it is generally interpreted as a localization satellite ${ }^{8}$, and it is absent in $U$ metal, where the $5 f$ electrons are well delocalized. It has also been observed in $U$ intermetallics ${ }^{9}$ where the $5 f$ bandwidth is decreased relative to $U$ metal but where the 5 electrons are still delocalized; in this case it is interpreted as a precursor effect to localization. In our study we also find the satellite to be absent in the thick $\mathrm{U}$ overlayer, where the $5 \mathrm{f}$ electrons are well delocalized, and we find it growing with decreasing $U$ dosage, i.e., when the $5 f$ bandwidth decreases. However, we find it decreasing again for very low $U$ dosage, where the $5 f$ electrons are clearly localized. It could be that for intermediate coverages the satellite superimposes onto the asymmetric tail of the $4 \mathrm{fmain}$ peak and therefore tends to be emphasized but, on the other hand, it is also clearly seen in bulk $\mathrm{UPd}_{3}{ }^{8}$, where the $4 \mathrm{f}$ levels are symmetrical. It seems to be most pronounced at the threshold of localization.

Figure 6 shows UPS valence band spectra of $U$ overlayers deposited on a Pd substrate at room temperature. At low dosages the signal at $\mathrm{E}_{\mathrm{F}}$ decreases with increasing $\mathrm{U}$ concentration, which points to a strong bonding interaction between $\mathrm{U}$ and $\mathrm{Pd}$. The $\mathrm{Pd}$ band narrows and the Pd4d intensity close to $E_{F}$ is suppressed: such findings can be understood in terms of filling of the $\mathrm{Pd} 4 \mathrm{~d}$ band and the dilution of $\mathrm{Pd}$ in a surface $\mathrm{U}$ matrix. At low coverage a small peak appears at $1 \mathrm{eV} \mathrm{BE}$, which has also been observed in $\mathrm{UPd}_{3}$, and is attributed to the localized $5 \mathrm{f}$ level. At this point the $5 f$ electrons in $U$ seem to be localized. The $1 \mathrm{eV}$ peak is very small, however, and would not be sufficient by itself to argue for $5 f$ localization. It is the coincidence of the (stronger) U4f XPS evidence and the UPS data which allows us to make such a statement. Resonant photoemission experiments should be performed on this system. They should reveal the continuous transition from localization to delocalization. A similar experiment has been done on the ternary $\mathrm{UPd}_{\mathrm{x}} \mathrm{Pt}_{3-\mathrm{x}}$ system ${ }^{10}$. At higher $\mathrm{U}$ dosage, the intensity at $\mathrm{E}_{\mathrm{F}}$ increases again 
due to $5 \mathrm{f}$ electrons which now become delocalized. This accompanies the development of the U4f asymmetry in Figure 4. At very high dosage the Pd4d peak becomes narrow and symmetrical and any asymmetry or non Gaussian-Lorentzian shape, which would suggest an initial state effect (band broadening) disappears. In addition, the shape of the $\operatorname{Pd} 4 d$ peak becomes identical in HeII and HeI while for Pd metal it differs because of matrix element effects. All this is suggestive of $\mathrm{Pd}$ atoms being embedded in a $\mathrm{U}$ matrix where they behave as atomically dispersed impurities. Such a system can be expected to be unstable and annealing experiments confirm this.

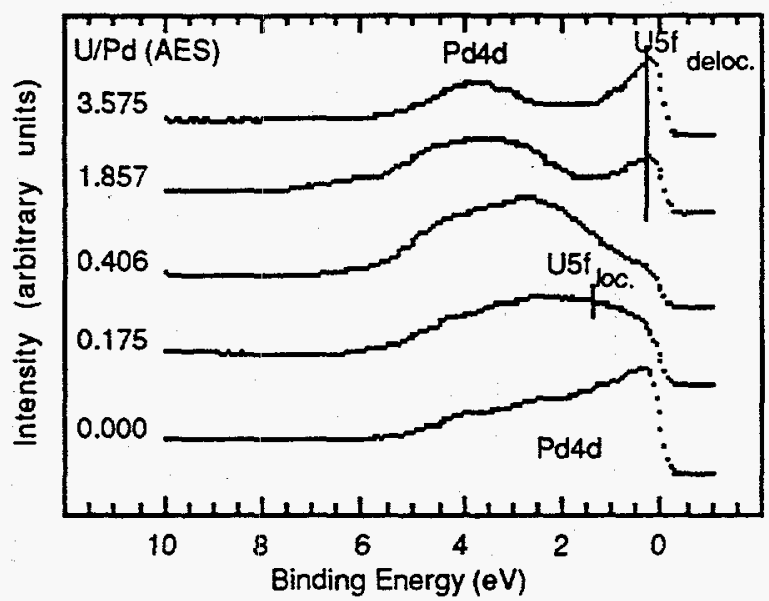

Fig. 6 UPS-Hell study of $U$ deposition on Pd. At low dosage a small peak is detected at about $1 \mathrm{eV} B E$ which is attributed to the localized $5 f$ emission.

At elevated temperatures the thick U overlayer reacts with Pd. This is shown by UPS-VB and XPS-U4f studies (Figures 7 and 8, respectively). In UPS, annealing at $200^{\circ} \mathrm{C}$ results in the decrease of the signal at the Fermi-level and in the transient appearance of a small peak at $0.8 \mathrm{eV}$ $\mathrm{BE}$. One might argue that this peak is due to the broadening of Pd4d superimposing on the shrinking U5f signal at $\mathrm{E}_{\mathrm{F}}$. However, we found that a decrease of $\mathrm{IEF}_{\mathrm{F}}$ by $60 \%$ in UPS corresponds to a decrease of the actual surface concentration of $U$ by less than $20 \%$ and conclude that the U5f emission in UPS does not disappear but actually shifts away from $E_{F}$. This together with the increase of the intensity at $0.8 \mathrm{eV}$ indicates that the $5 \mathrm{f}$ emission shifts to that location, i.e., the U5f electrons become localized (this interpretation is corroborated by the XPS study, see below): the excess surface $U$ reacts with Pd to form UPd, where the U5f electrons are localized. At higher temperatures, the $5 f$ peak disappears completely because of the bulk diffusion of $U$ (see also Section 4). Notice that the Pd VB broadens during annealing and that the most notable change occurs between RT and $200^{\circ} \mathrm{C}$, indicating U reaction with Pd. The UPS spectrum at $200^{\circ} \mathrm{C}$ indeed bears strong similarities with XPS-VB spectra of $\mathrm{UPd}_{3}$. Such strong variation of the electronic structure and the low temperature where it occurs demonstrate the very high reactivity of the system (TM-rare earth systems often have to be annealed at $400^{\circ} \mathrm{C}$ to $600^{\circ} \mathrm{C}$ to 
complete the reaction with the substrate ${ }^{11}$ ). XPS data complete our picture of the annealing induced surface reaction (Figure 8). Annealing results in the narrowing of the $4 \mathrm{f}$ emission and in its shift to higher BE: the non uniform surface phase, which was responsible for the U4f broadening (see discussion of Figure 4), reacts with the Pd substrate to form a single uniform near-surface alloy, thus the narrowing of the U4f (Figure 9). The surface $U$ atoms change their electronic structure away from that of U metal (low BE) to that of a U-Pd alloy, thus the shift to high BE. We actually see a reversal of the shift to low BE observed with increasing $U$ dosage (Figure 4) because annealing results in the dilution of $U$ in Pd. In addition, the U4f peak becomes more symmetric after annealing indicating a decrease of the DOS at $E_{F}$ : the $5 f$ electrons become localized, consistent with the formation of UPd3. The phase diagram shows $\mathrm{UPd}_{3}$ to be the U-Pd alloy with the highest $\mathrm{U}$ concentration that is stable as a bulk phase. Therefore, we can expect a $U$ overlayer or $U$ accumulation region to react to form this alloy. (We must point out, however, that in the near-surface region alloys may exist which are not stable in the bulk phase, therefore the bulk phase diagrams must be considered as a guide rather than a proof.) AES studies (section 4) will confirm the formation of UPd 3 by a low temperature diffusion-reaction.

Let us summarize our findings for the U-deposition of Pd. U strongly interacts with Pd. At low dosage it does not agglomerate in clusters but atomically disperses on the Pd surface. At higher dosages it accumulates on the surface and, driven by the instability of such a system, begins to spontaneously diffuse into the Pd substrate. This interdiffusion is enhanced by gentle annealing and its driving force is the transformation of the $U$ accumulation layer into UPd3, which is the U$\mathrm{Pd}$ compound with the highest possible $\mathrm{U}$ concentration. Localization of the $5 \mathrm{f}$ electrons is observed for the dispersed U surface layer (high dilution) and for the UPd 3 near surface alloy.

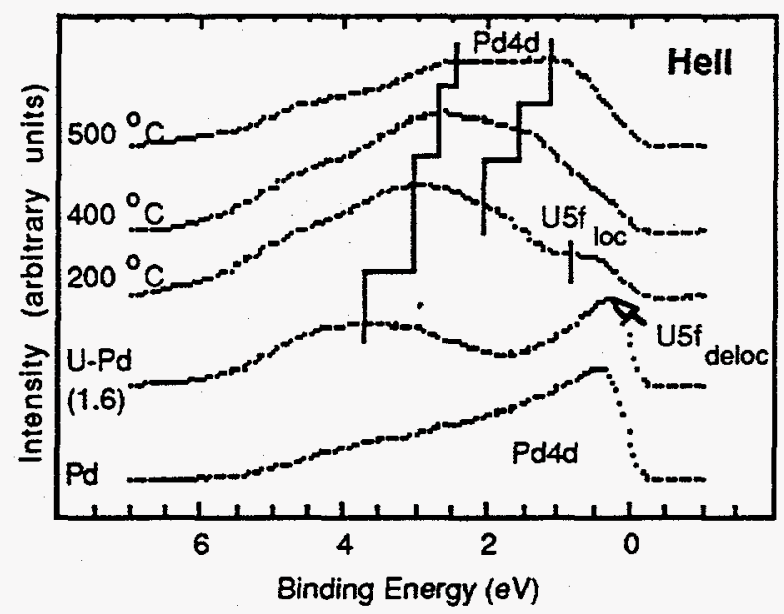

Fig. 7 Hell study of the annealing of U-Pd. Sharp drop of the signal at $E_{F}$ and broadening of the Pd4d point to the reaction between $U$ and $P d$ even at $200^{\circ} \mathrm{C}$. 


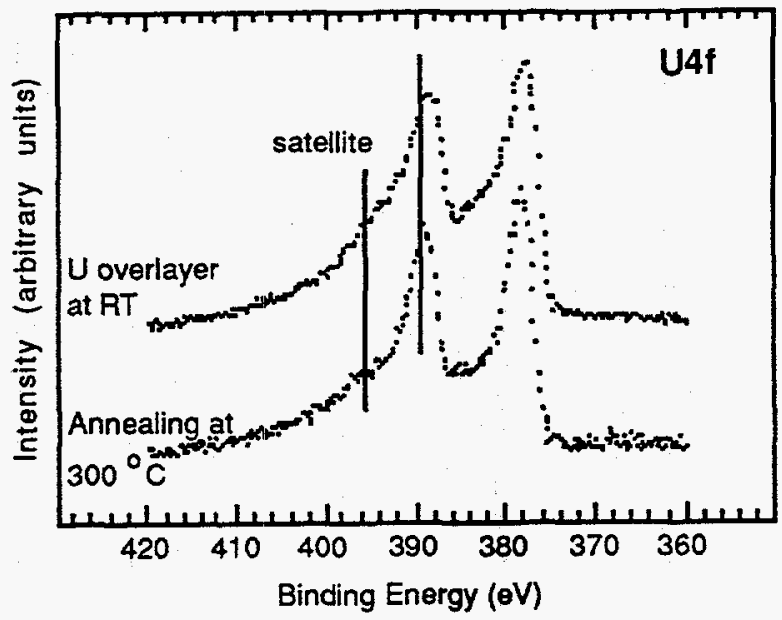

Fig. 8 U4t study of annealing U-Pd.

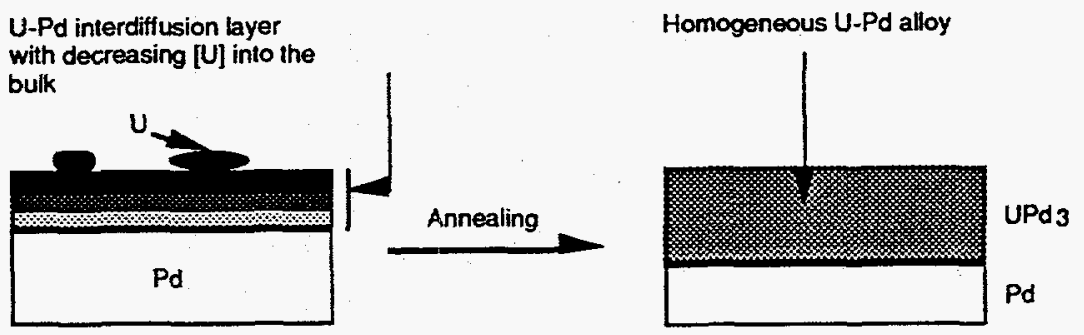

Fig. 9 Low temperature diffusion reaction of $U$ with Pd results in the formation of a homogeneous near surface alloy putting $\mathrm{U}$ in one well defined chemical environment.

We made a comparable study for the U-Pt system. As we stated above, there is no U-Pt bulk alloy with localized $5 \mathrm{f}$ electrons, but there is the heavy-fermion UPt 3 where the bandwidth of the $5 f$ is very narrow, i.e., the $5 f$ electrons are close to localization. Thus our hope was that supplementary band narrowing at the surface would induce localization. Figure 10 shows a UPS-HeII study of the U-Pt system with increasing U dosages.

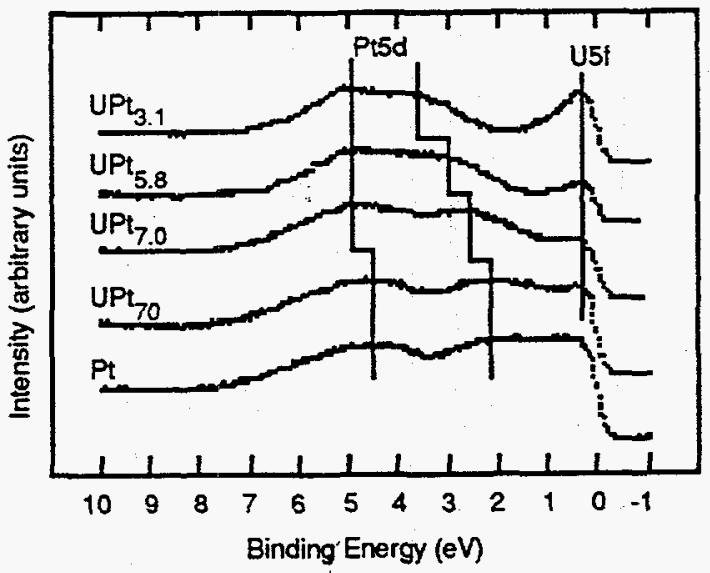

Fig. 10 UPS-Hell study of $U$ deposited on $P t$ at room temperature. 
The $\mathrm{U}$ coverage was determined by the ratio of the $\mathrm{U} 4 \mathrm{f} / \mathrm{Pt} 4 \mathrm{f}$, with an information depth of about 8 monolayers (for a thin overlayer this overemphasizes the Pt signal, therefore the apparent higher $\mathrm{U}$ dilution when compared to U-Pd, where the information depth was about 3 monolayers). The narrowing of the Pt5d band with increasing $U$ coverage indicates a strong interaction between $\mathrm{U}$ and $\mathrm{Pt}$ and near surface diffusion of $\mathrm{U}$ into Pt. At low dosages $\mathrm{U}$ deposition results in the decrease of the intensity at $\mathrm{E}_{\mathrm{F}}$ when compared to the Pt5d valence band, demonstrating again the strong bonding between $\mathrm{U}$ and Pt. This decrease is less marked than for the UPd system and there is no signal at $1 \mathrm{eV}$. Instead, the intensity comes back at the Fermilevel for higher dosages: UPS does not give any indication for $5 f$ localization. The core levels are asymmetric for all $U$ coverages, which confirms that the $5 f$ electrons are delocalized, even for dilute systems.

In the U-Pt system annealing results in the bulk diffusion of U. UPS data show the Pt valence band to broaden and the upper Pt5d band to shift to its position in pure Pt metal (Figure 11). The 5f signal at the Fermi-level decreases, but the decrease is more gradual than in U-Pd and there is no signal at $1 \mathrm{eV}$. We conclude that as in U-Pd, annealing results in the dilution of a U surface accumulation layer, but that alloy formation does not lead to $5 \mathrm{f}$ localization. This was actually to be expected because U-Pt bulk alloys do not have localized 5 f electrons. The only situation where we could have expected to see $5 f$ localization was at low U coverage, but the experiment shows that even under those circumstances the $5 \mathrm{f}$ electrons are delocalized.

Figure 12 compares the UPS-HeII data for several other systems we investigated. We must caution that the $\mathrm{Au}$ and $\mathrm{Cu}$ data have been taken on substrates that were not annealed after. sputtering and, therefore, have a high concentration of surface defects. (This probably enhances the reactivity of the substrate and may result in near surface diffusion of $U$ through cracks and surface defects.) The graphite substrate shows a signal at $3 \mathrm{eV}$ which comes from the Mo holder, and $\mathrm{O}$ contamination at $6 \mathrm{eV}$ (these were very early data!). The $3 \mathrm{eV}$ signal was removed by subtraction of the graphite (and Mo) background taken before $U$ deposition leaving a symmetric $\mathrm{O} 2 \mathrm{p}$ peak that we found for $\mathrm{UO}$ or $\mathrm{U}$ oxycarbide. $\mathrm{Au}, \mathrm{Cu}$, and graphite substrates have the advantage of not having d-emission in the region of interest $(0-2 \mathrm{eV})$. The $\mathrm{U}-\mathrm{Au}$ and $\mathrm{U}-\mathrm{Cu}$ systems show that even for small $U$ concentrations, of the order of 0.3 monolayers, the U5f signal stays right at the Fermi-level, i.e., the $5 f$ electrons are delocalized. There is a stronger interaction between $U$ and $A u$ than between $U$ and $\mathrm{Cu}$ as shown by the narrowing of the Ausd band, while the $\mathrm{Cu} 3 \mathrm{~d}$ band changes very little. A high-resolution spectrum taken on a highly diluted $\mathrm{U}$ overlayer on graphite shows a peak at about $0.8 \mathrm{eV}$ below the Fermi-level (Figure 13). 


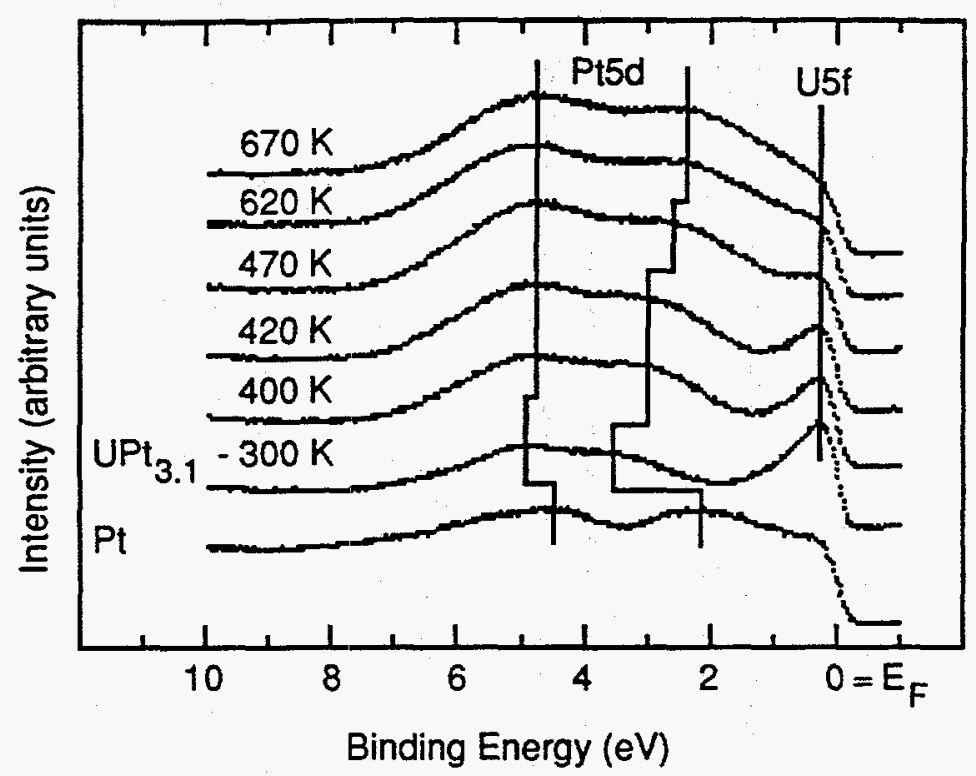

Fig. 11 UPS-Hell study of annealing of U-Pt. Annealing leads to the decrease of the surface concentration of U and U-Pt alloy formation.

Again there is $\mathrm{O}$ and Mo contamination present, but neither is thought to be capable of producing the $0.8 \mathrm{eV}$ signal: we extensively studied $\mathrm{UO}$ and $\mathrm{UO}_{2}$ (Section 3) and never found any indication for a peak at $0.8 \mathrm{eV}$, but instead found the $5 \mathrm{f}$ signal either right at $\mathrm{E}_{\mathrm{F}}(\mathrm{U}, \mathrm{UO})$ or between $1.2-2.2 \mathrm{eV}\left(\mathrm{UO}_{2}\right)$. The Mo signal is very weak even compared to the $1 \pi$ and $5 \sigma$ emissions of graphite showing less than $2 \%$ of the surface covered is Mo. Thus, there is not enough Mo to produce such a satellite even if it was to occur in a U-Mo compound. Also the signal seems to be too sharp to be produced by a graphite emission. However, the measurements should be repeated on a clean sample without $O$ and Mo contamination to verify the validity of these arguments. At this point we believe the $0.8 \mathrm{eV}$ signal to be a genuine characteristic of the U-graphite system. It could be due to a localization (correlation) effect occurring in very small $U$ clusters, but we should also mention that it has been claimed recently that $U$ deposits on graphite forming a $\beta$-phase ${ }^{12}$. The enlarged $U$ lattice constant in this $\beta$-phase and the decreased $\mathrm{f}-\mathrm{f}$ overiap could also play a role in producing the satellite (it is not clear, however, where the Ugraphite system would acquire the thermodynamic driving force to form beta-U because graphite interacts only weakly with $U$; this is why clusters form spontaneously). Weak interaction between $U$ and graphite is also manifested by the fact that annealing at $800^{\circ} \mathrm{C}$ does not induce diffusion, while for $\mathrm{Pd}$ or $\mathrm{Pt}$ it starts already at $200^{\circ} \mathrm{C}$.

From these findings we conclude that at the dosages we used, which were always above 0.2 monolayers, the U5f electrons are delocalized, except for Pd where they are also localized in the bulk compound and perhaps for graphite where U deposits as clusters. Maybe U5f localization 
will be observed at even lower $U$ concentrations, but such experiments would involve synchrotron radiation studies to get better statistics and, in the case of d-metal substrates, to separate a weak U5f signal from the intense substrate background using resonant photoemission.

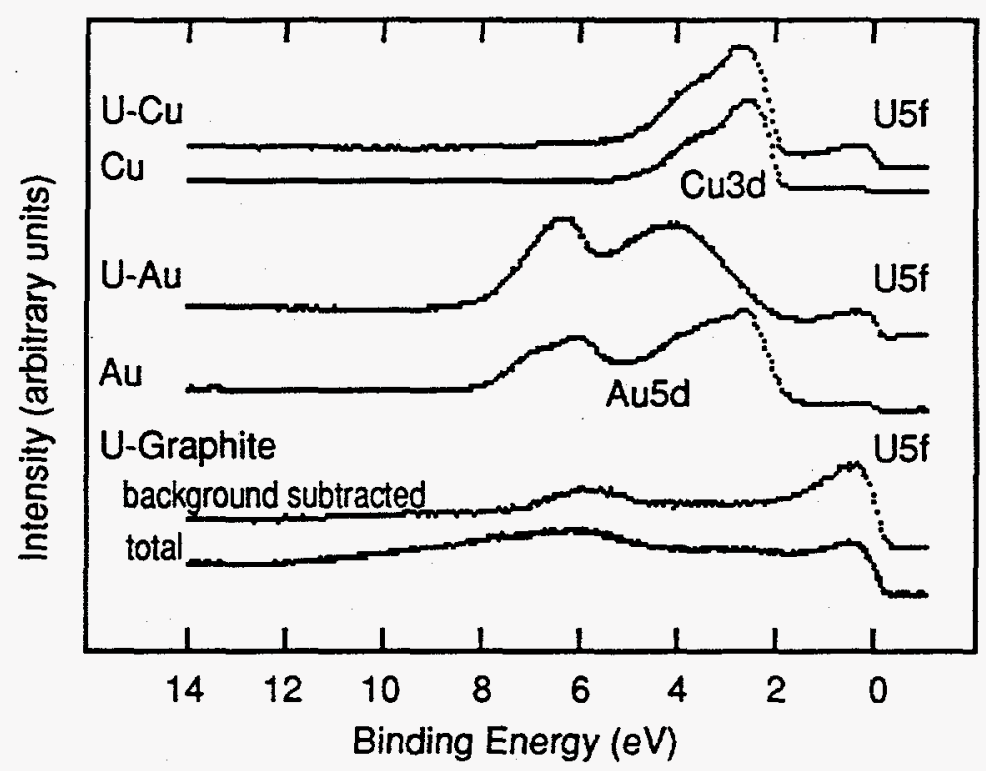

Fig. 12 UPS-Hell spectra for $\mathrm{U}$ submonolayers on $\mathrm{Au}, \mathrm{Cu}$ and graphite (about 0.3 monolayers).

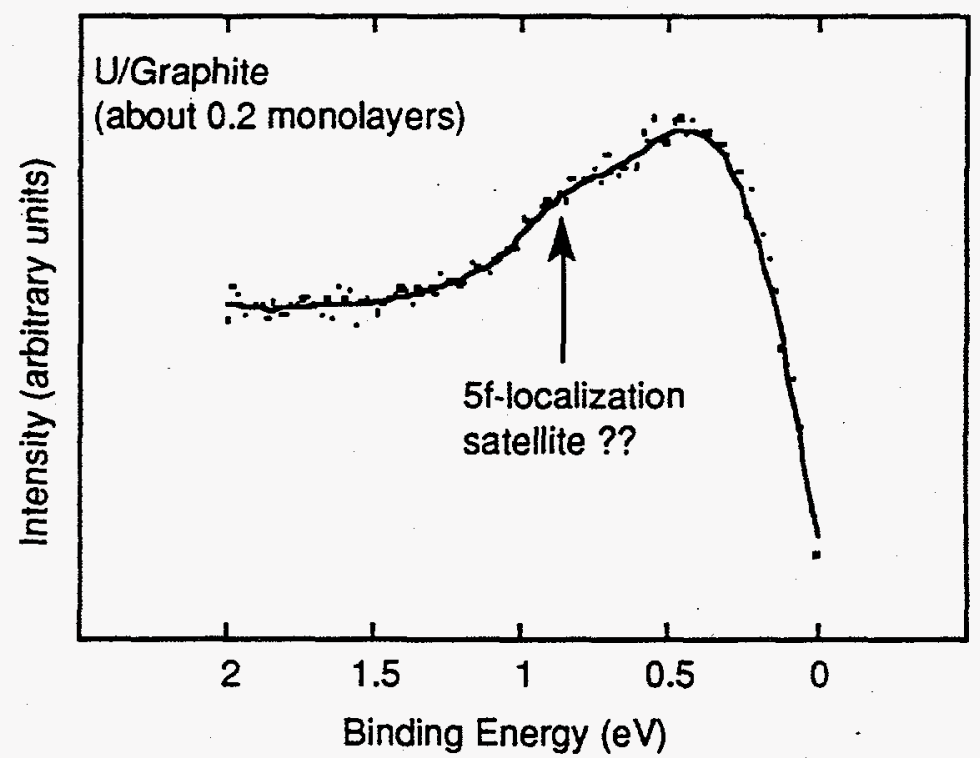

Fig. 13 UPS-Hell spectrum ("high resolution" / DPCMA) of U overlayer on graphite. The spectrum is cut before the Fermi-level. 


\section{Surface Reactions on Thin U Overlayers}

After characterization of the clean U-substrate systems we investigated their reactions with gases. With these studies we want to contribute to a deeper understanding of the reactivity of the actinide-substrate interface with, as main application, the handling and disposal of nuclear waste. We followed the evolution of the surface reactivity with increasing $U$ concentration, i.e., from the low concentration limit, where the $U$ atoms are dispersed on the substrate, to the formation of an interface at high concentration. We emphasized the following three reaction systems:

a) Reaction of a $\mathrm{U}-\mathrm{TM}$ interface (high $\mathrm{U}$ concentration) with a reactive oxidant $\left(\mathrm{O}_{2}\right)$. Our aim was to follow the corrosive decomposition of the interface.

b) Reaction of $U$ deposited on an inert substrate (graphite) limiting the actinide phase to the information depth of surface spectroscopies. This setup allows the study of terminal reaction products.

c) Reaction of finely dispersed $U$ on a TM substrate (low $U$ concentration) with a low reactivity gas $(\mathrm{CO})$. In this setup, $U$ loses most of its own reactivity but changes the adsorption properties of the substrate; it takes the roles of a promoter or poison (application: catalysis).

\section{a. Corrosion-Induced Interface Decomposition}

We studied the behavior of several interfaces (U-Pd, U-Pt, U-Si) in the presence of $\mathrm{O}_{2}$. In all cases $\mathrm{U}$ shows a high affinity for oxygen and $\mathrm{O}_{2}$ adsorption generally results in the decomposition of the $U$-substrate interface and the segregation of $U$ to the surface where it forms $\mathrm{UO}_{2}$. Figure 14 shows a $\mathrm{HeII}$ study of $\mathrm{O}_{2}$ adsorption on a U-Pt surface. $\mathrm{O}_{2}$ adsorption results in the intensity increase of a broad and asymmetric $\mathrm{O} 2 \mathrm{p}$ signal in the region of $3-0 \mathrm{eV}$, which is typical for oxidic oxygen in $\mathrm{UO}_{2}$. The signal at the Fermi-level, which we attributed to the $5 \mathrm{f}$ electrons of metallic $\mathrm{U}$, decreases and a peak appears at about $1.8 \mathrm{eV}$. This peak is also observed in $\mathrm{UO}_{2}$ where it is assigned to the localized $5 \mathrm{f}^{2}$ level. These findings clearly show the surface of $\mathrm{U}$ to be oxidized to $\mathrm{UO}_{2}$, and this is confirmed by the XPS-U4f data. In addition, surface oxidation is accompanied by the broadening of the Pt5d band, whose upper sub-band moves towards the Fermi-level. This is explained by the breaking of U-Pt bonds, which is responsible for the narrowing of the Pt5d after $\mathrm{U}$ deposition (Figure 10). Notice that at high $\mathrm{O}_{2}$ dosage there is no $\mathrm{Pt}$ signal left even though there was a significant signal after $\mathrm{U}$ deposition. This shows $\mathrm{O}_{2}$ adsorption to result in the surface segregation of $\mathrm{U}$ where it forms a continuous $\mathrm{UO}_{2}$ overlayer 
(Figure 15). Above $10 \mathrm{~L} \mathrm{O}_{2}$ the $\mathrm{U} 5 \mathrm{f}^{2}$ emission shifts to lower $\mathrm{BE}$ due to the transformation of $\mathrm{n}$-type substoichiometric $\mathrm{UO}_{2-\mathrm{x}}$ into $\mathrm{p}$-type stoichiometric or hyperstoichiometric $\mathrm{UO}_{2+\mathrm{x}}$ resulting in the decrease of the Fermi-energy.

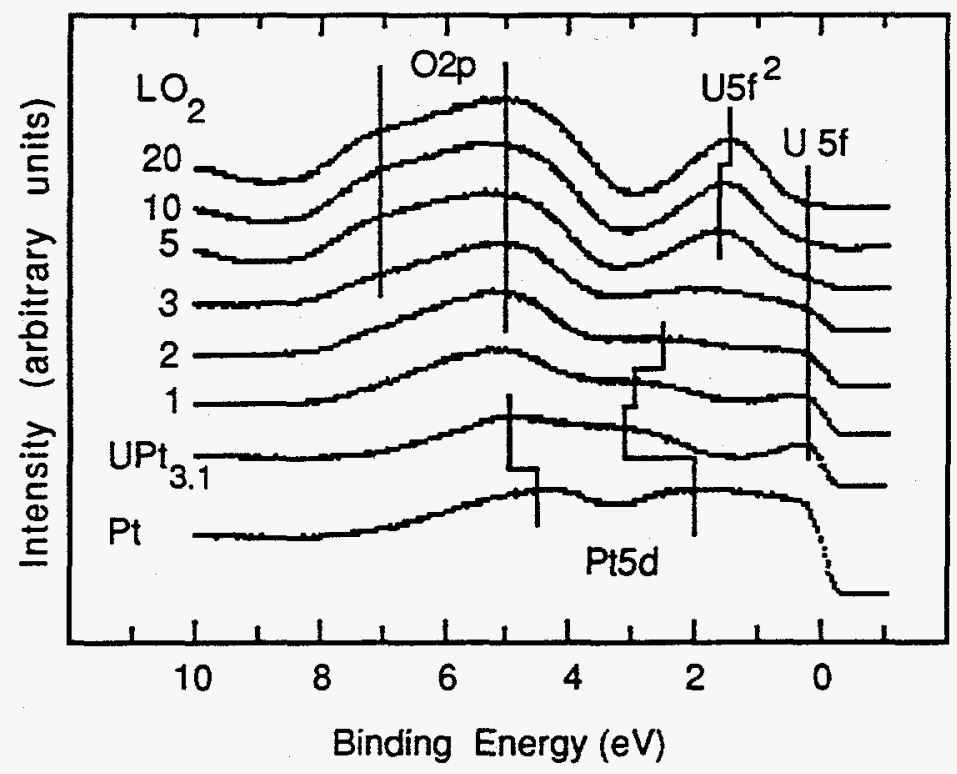

Fig. $14 \mathrm{O}_{2}$ adsorption on U-Pt at room temperature. All $U$ spontaneously oxidizes and the surface becomes covered by a $\mathrm{UO}_{2}$ layer.

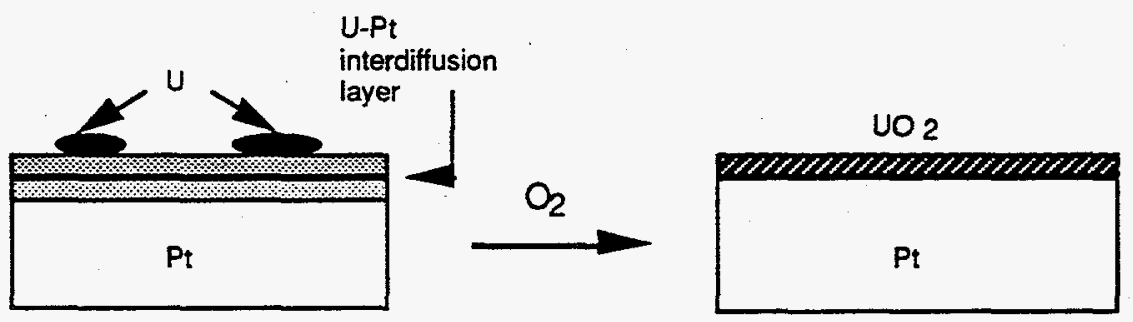

Fig. $15 \mathrm{O}_{2}$ adsorption on a $U$ overlayer on $P t$ leads to the oxidation $U$. The U-Pt interface (interdiffusion layer) decomposes, $U$ segregates to the surface and the $\mathrm{Pt}$ substrate becomes covered by a continuous $\mathrm{UO}_{2}$ film.

U-Pd shows similar behavior. Figure 16 displays the changes of the Pd3d core-level spectra with $U$ oxidation. $U$ deposition results in a chemical shift of the Pd3d to higher BE because of the U-Pd interaction. Most of the Pd3d signal is shifted indicating that the interdiffusion layer is several atomic layers deep. With $\mathrm{O}_{2}$ adsorption the Pd3d shifts back to lower $\mathrm{BE}$ and eventually back to its position in pure Pd metal. The continuous shift is consistent with the gradual depletion of $U$ in the interdiffusion layer. The absence of the Pd3d signal of Pd oxide, at $1 \mathrm{eV}$ higher $\mathrm{BE}$ than the metal, shows that up to $60: \mathrm{L} \mathrm{O}_{2} \mathrm{Pd}$ does not oxidize, probably because it is protected by a $\mathrm{UO}_{2}$ overlayer (Pd has a low affinity for oxygen anyway). 
We also studied the oxidation behavior of $U$ on graphite. Graphite is an inert substrate on which $\mathrm{U}$ was shown to deposit as clusters. Our STM group claims to have observed a strong decrease of $U$ reactivity in these clusters: $U$ would not oxidize at ambient pressure for about $30 \mathrm{~min}$., as shown by a tunnel current proving $U$ to be metallic ${ }^{3}$. We believe that at low $U$ concentrations and in the presence of $\mathrm{CO}_{2}$, hydrocarbons, nitrogen, etc. a thin layer of metallic $\mathrm{UO}$, which is stabilized by UC, US, UN,... ("dirty oxide"), is formed. This would account for the observed tunneling current. A UHV experiment of the $\mathrm{O}_{2}$ adsorption on a thin layer of $\mathrm{U}$ on graphite, at room temperature, indeed shows an almost unchanged high reactivity of the surface (Figure 17). Within an $\mathrm{O}_{2}$ dosage of $5 \mathrm{~L}$ most of the surface $\mathrm{U}$ is oxidized to $\mathrm{UO}_{2}$ !

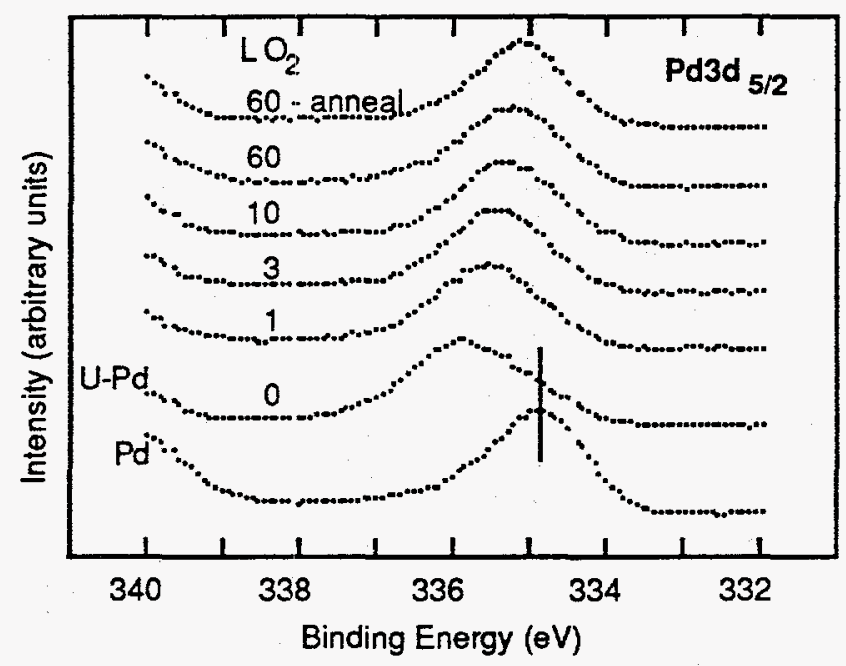

Fig. 16 Pd3d with $U$ adsorption and oxidation. The initial shift to high $B E$ after $U$ adsorption is reversed after oxidation of $U$ which points to the corrosive decomposition of the U-Pd interdiffusion region.

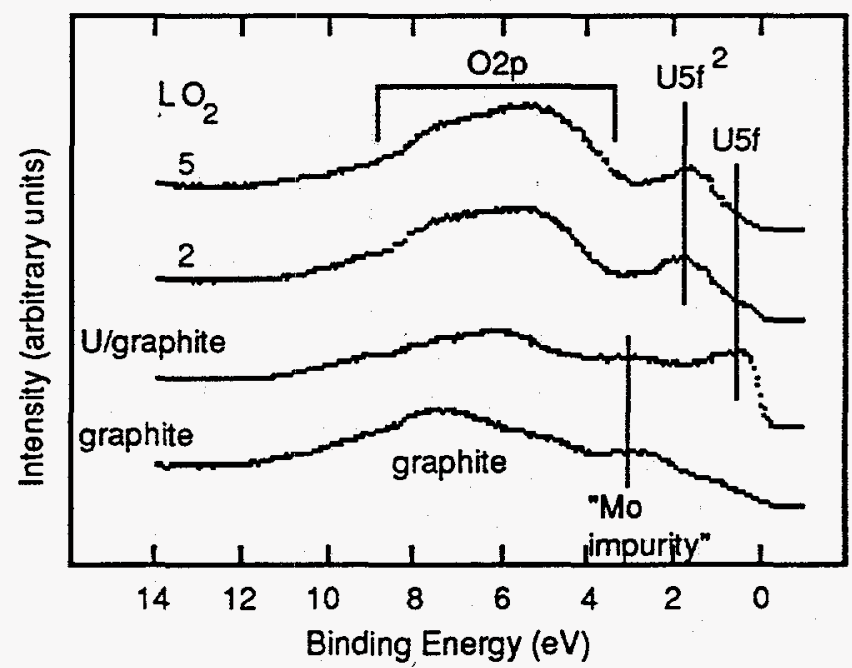

Fig. 17 Hell study of the oxidation of a thin $U$ overlayer (about 0.3 monolayer) on graphite. $U$ shows a high affinity for oxygen and exposure to $5 \mathrm{LO}_{2}$ results in the almost complete oxidation of all surface $\mathrm{U}$. 
The U-Si system shows a slightly different reactivity towards oxygen. We deposited U on a ptype $\mathrm{Si}(111)$ single crystal. We did not discuss the U-Si system in Section 2 because the Si substrate was not pure (p-doping) thus introducing some unknown effect. Let us briefly discuss it here. A U overlayer interacts with $\mathrm{Si}$ as seen by the shift of the Si2p to lower BE (Figure 18), which may be explained by the formation of a Schottky-barrier. Rare earths deposited on $\mathrm{Si}$ substrates actually build up a weak Schottky-barrier ${ }^{13}$. The U4f emission is sharp, even for a medium $\mathrm{U}$ dosage (for Pd and $\mathrm{Pt}$ a broad $4 \mathrm{f}$ peak was produced), which might be explained either by the absence of an interdiffusion layer or by the absence of a chemical shift for $U$ in the interdiffusion layer (Figure 19). Annealing at $400^{\circ} \mathrm{C}$ leads only to a weak decrease of the $U$ signal, while in $\mathrm{Pd}$ and $\mathrm{Pt}$ the U4f signal was already attenuated at $200^{\circ} \mathrm{C}$, suggesting a weaker interaction between $U$ and $S i$ than $U, P d$, or Pt. The intense satellite at $6 \mathrm{eV} \mathrm{BE}$ could be interpreted as a precursor to localization, but a clear assignment is complicated by the fact that $\mathrm{U}-\mathrm{Si}$ has a plasmon peak at $6 \mathrm{eV}$ (the satellite is at least partially due to a plasmon loss). UPSVB spectra did not show any evidence for $5 \mathrm{f}$ localization (a correlation satellite at about $1 \mathrm{eV}$ is missing completely). $\mathrm{O}_{2}$ adsorption results in the partial oxidation of $\mathrm{U}$ to $\mathrm{UO}_{2}$ as shown by the increase in intensity of the U4f oxide peak at $3 \mathrm{eV}$ higher $\mathrm{BE}$ than the metal peak. In contrast to the $\mathrm{Pd}$ and $\mathrm{Pt}$ systems, the Si substrate itself has a high $\mathrm{O}$ affinity. The development of a peak at $102 \mathrm{eV} \mathrm{BE}$ (Figure 19), characteristic of $\mathrm{SiO}_{2}$, shows that $\mathrm{Si}$ oxidizes even at $\mathrm{O}_{2}$ dosages where part of the $U$ is still metallic.

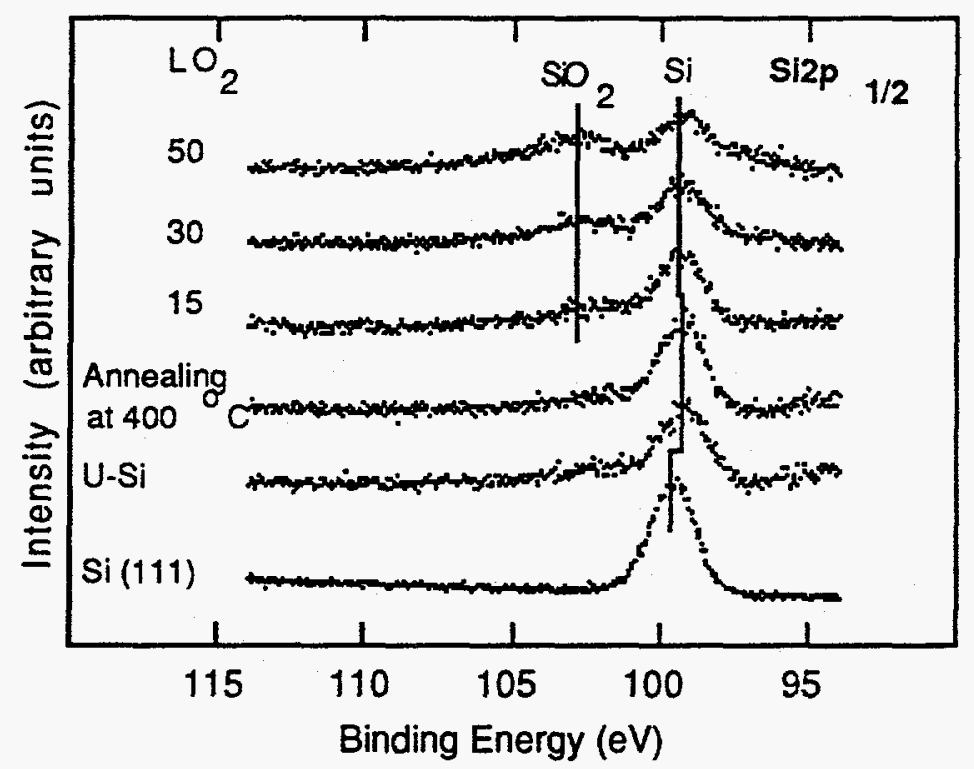

Fig. 18 Evolution of the Si2p emission with $\mathrm{U}$ coverage and oxidation. $\mathrm{O}_{2}$ adsorption results in a partial oxidation of Si to $\mathrm{SiO}_{2}$. 


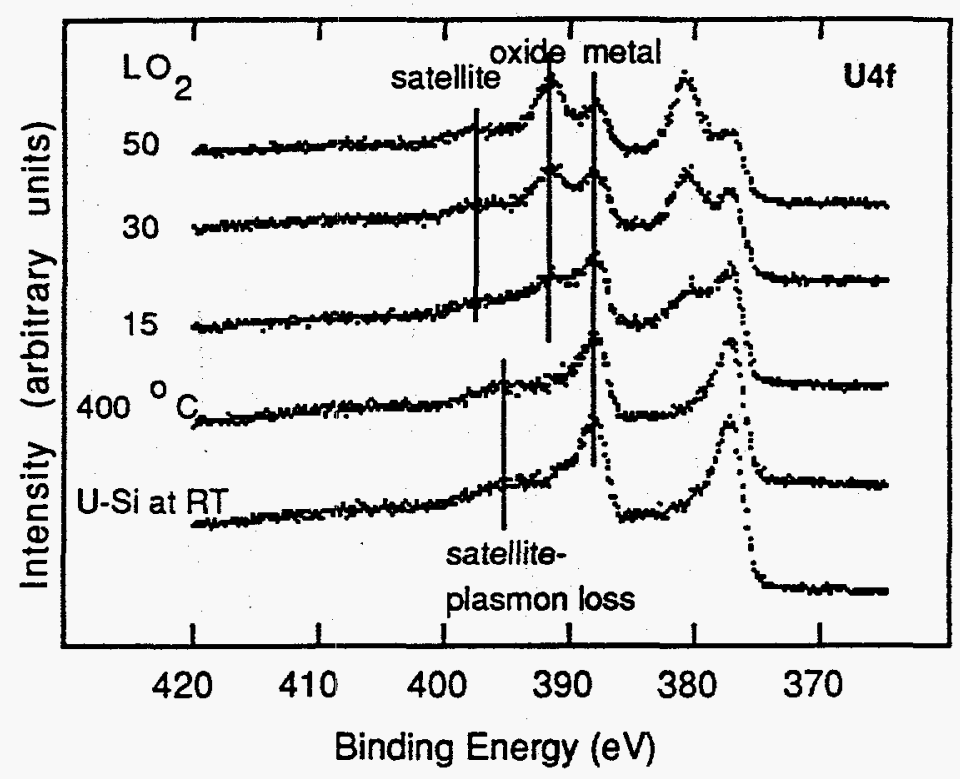

Fig. 19 Evolution of the U4f emissions with $U$ coverage and oxidation.

The $\mathrm{O}_{2}$ adsorption study shows that $\mathrm{U}$-substrate interfaces always decompose spontaneously in the presence of $\mathrm{O}_{2}$, and that in all cases $U$ segregates to the surface. These surface reaction studies are useful when combined with the bulk diffusion study of Section 4 , where we follow the diffusion behavior of $U$ for varying degrees of surface oxidation.

\section{b. Use of Thin Layers to Study Terminal/Oxidation Reaction Products}

The study of the formation and stability of the final reaction products is of importance both in corrosion and in catalysis research. Unfortunately, under UHV conditions, where the sample can be exposed only to relatively minor amounts of gases, the bulk of a reactive sample such as $U$ often acts as a reaction product sink: it continuously abstracts adsorbed gas atoms $(\mathrm{O}, \mathrm{C}, \mathrm{H}, \ldots)$ from the surface reaction layer until it becomes saturated, which is difficult to achieve under UHV conditions. It thereby impedes the formation of terminal reaction products, e.g., high oxidation states. This is especially disadvantageous at high temperatures, where diffusion allows surface atoms to migrate rapidly into the bulk. Thin layers of $U$ on an inert substrate such as graphite provide an easy solution because the $U$ bulk is eliminated. We shall discuss one application of this below.

We used thin layers of $U$ on graphite to assess the thermodynamic stability of $U$ oxycarbide surface layers $\left(\mathrm{UO}_{\mathrm{x}} \mathrm{C}_{1-\mathrm{x}}\right)$ when compared to $\mathrm{UO}_{2}$. The oxycarbide is a solid solution of $\mathrm{UO}$ and $\mathrm{UC}$ and may be viewed as carbon-stabilized UO. We are interested in UO because it is a U oxide 
with delocalized U5f electrons which may have some applications in catalysis (see next paragraph). We previously produced it as an overlayer on $U$ metal by adsorption of $\mathrm{CO}^{14}$. We wanted to determine whether $\mathrm{UO}$ is only a transient species in the formation $\mathrm{UO}_{2}$. (UO seems to be stable under UHV conditions because CO does not adsorb on a UO overlayer, and therefore, the reaction stops once the $U$ metal is covered by a UO monolayer) or whether it is thermodynamically stable when compared to $\mathrm{UO}_{2}$. In the latter case it should be possible to convert $\mathrm{UO}_{2}$ into $\mathrm{UO}$ by exposing a $\mathrm{UO}_{2}$ overlayer to a carbon source such a $\mathrm{C}_{2} \mathrm{H}_{4}$. The question we ask is: can chemical reaction (1) take place at moderate (catalytically realistic) temperatures?

$$
\mathrm{U}+\mathrm{UO}_{2}+2 \mathrm{C} \stackrel{300^{\circ} \mathrm{C}}{\longrightarrow} 2 \mathrm{UOC}
$$

We do not know what happens to hydrogen in such a reaction but it is conceivable that it reacts with chemisorbed $\mathrm{C}_{2} \mathrm{H}_{4}$ to form $\mathrm{C}_{2} \mathrm{H}_{6}$ or that it desorbs as $\mathrm{H}_{2}$. Experimental results are shown in Figures 20 and 21. We deposited a thin layer (about 8 monolayers) of $U$ on graphite. $U$ forms a continuous overlayer (the system is beyond the cluster growth stage) as shown by the near absence of the $\mathrm{C}$ 1s signal. We partially oxidized the system by adsorbing $\mathrm{O}_{2}$, which leads to the growth of the $\mathrm{UO}_{2}$ signal as shown in the XPS (oxide-shifted U4f) and UPS data (asymmetric $\mathrm{O} 2 p$, localized $U 5 \mathrm{f}^{2}$, and some intensity left at the Fermi-level due to non-reacted $\mathrm{U}$ ). We then checked the stability of the oxide phase at elevated temperature. At this stage, thin layers behave differently than a bulk sample: for the latter the $O$ signal would begin to decline because of $O$ motion into the bulk, while only a slight decrease was observed for the thin layer system. At $300^{\circ} \mathrm{C}$ the difference is not too pronounced, but this changes at e.g. $900^{\circ} \mathrm{C}$ where $\mathrm{O}$ completely disappears. We finally exposed the surface to a $\mathrm{C}_{2} \mathrm{H}_{4}$ atmosphere $\left(\mathrm{P}_{\mathrm{C} 2 \mathrm{H} 4}=1 \times 10^{-4} \mathrm{~T}\right)$ to observe if the oxide would transform into the oxycarbide. The $\mathrm{U} 4 \mathrm{f}$ data show that the $\mathrm{UO}_{2}$ oxide signal disappears while a peak appears at slightly higher $B E$ than $U$ metal, which is broader than the $\mathrm{U}$ metal emission. In UPS the $\mathrm{O} 2 \mathrm{p}$ peak becomes symmetric, the $\mathrm{U} 5 \mathrm{f}^{2}$ emission of $\mathrm{UO}_{2}$ disappears, and the signal at the Fermi-level increases. (Annealing $\mathrm{UO}_{2}$ on bulk $\mathrm{U}$ metal leads to the decrease of the O2p signal which, however, keeps its asymmetric shape ${ }^{15}$.) All these findings strongly suggest the transformation of $\mathrm{UO}_{2}$ into $\mathrm{UO}$ : we previously observed that $\mathrm{CO}$ and $\mathrm{CO}_{2}$ adsorption on $\mathrm{U}$ results in the growth of a symmetric $\mathrm{O} 2 \mathrm{p}$ line and a missing $5 \mathrm{f}^{2}$ peak. The symmetry of the $\mathrm{O} 2 \mathrm{p}$ line was determined as one characteristic of $\mathrm{UO}$ distinguishing it from $\mathrm{UO}_{2}$, which has an asymmetric $\mathrm{O} 2 \mathrm{p}$. Furthermore, in $\mathrm{UO}_{2}$ the $5 \mathrm{f}$ electrons are localized and form the $5 \mathrm{f}^{2}$ level at about $1.4 \mathrm{eV}$, while in $\mathrm{UO}$ they are itinerant and situated at the Fermi-level. Hence both XPS and UPS show UO 2 to be replaced by a more reduced (small BE shift from the 


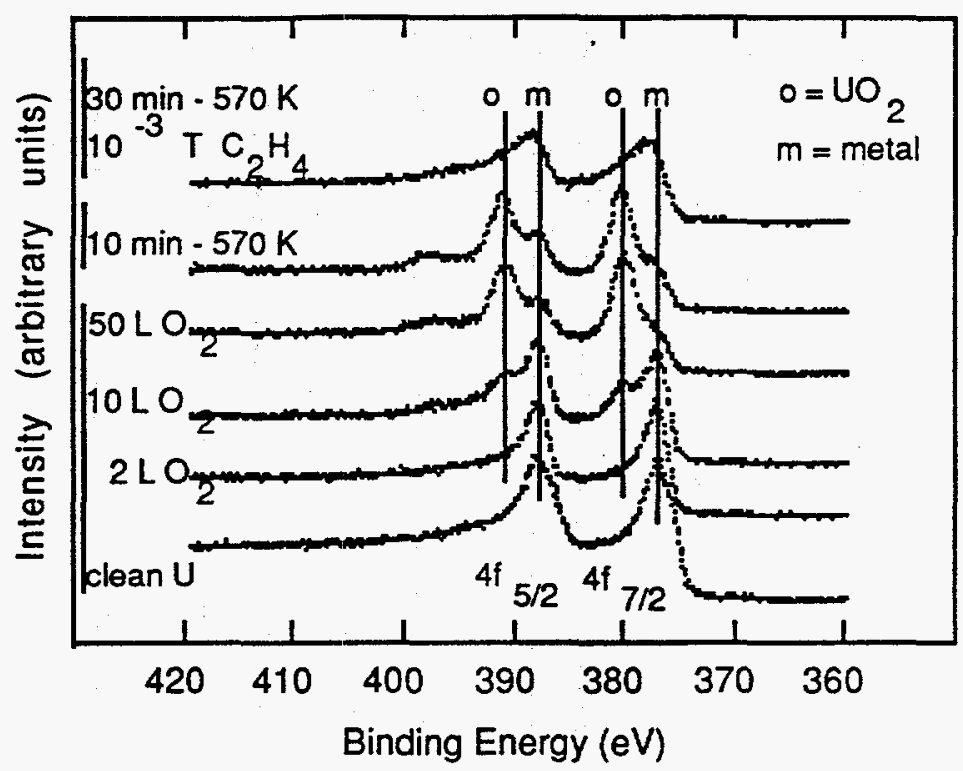

Fig. 20 XPS-U4f study of the transformation of $U$ into $\mathrm{UO}_{2}$ followed by the reaction of $\mathrm{UO}_{2}$ (and $\mathrm{U}$ metal) with $\mathrm{C}_{2} \mathrm{H}_{4}$ to form the oxycarbide.

U metal in XPS) metallic oxide intensity at $E_{F}$ in UPS also (O2p in UPS), which must be UO. Reaction is further evidenced by the decrease of the Ols peak and the simultaneous growth of the Cls signal, showing that part of the oxygen is replaced by carbon.

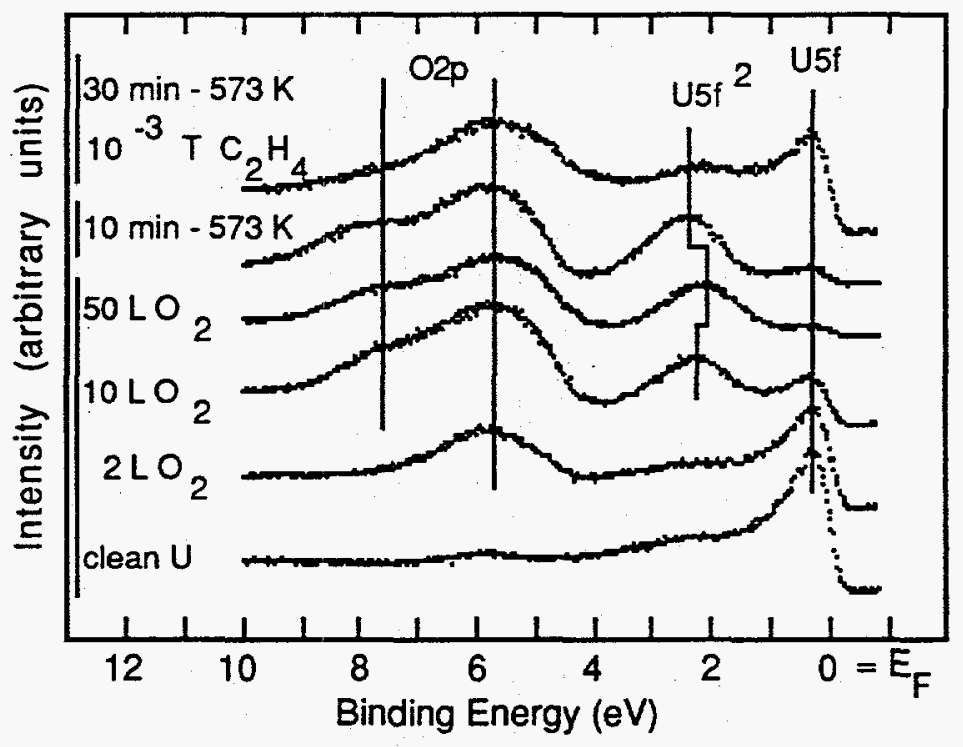

Fig. 21 UPS-Hell study of the transformation of $U$ into $\mathrm{UO}_{2}$ followed by the reaction of $\mathrm{UO}_{2}$ (and $U$ metal) with $\mathrm{C}_{2} \mathrm{H}_{4}$ to form the oxycarbide. Oxycarbide formation results in the increase of the intensity at $E_{F}$. 


\section{c. U-Doped Surfaces: Actinides as Promoters in Catalysis}

In the mid-80s several research groups investigated possible catalytic properties of actinides and actinide compounds (Karlsruhe, Strassbourg, Livermore, Namur). Different scientific ideas led to the choice of actinides as model catalysts (e.g., mixed valence, hydrogen storage, and actinide intermetallics as precursor materials for highly dispersed noble metal catalysts, etc.). We shall concentrate here on one case that mainly applies to metallic systems: actinides have highly correlated f-electrons and, in that respect, are similar to late transition metals (Ni, Pd, Pt, etc.) where the d-electrons are found in narrow bands. It has been argued that the catalytic properties of the late transition metals are due to an interplay between highly correlated d-electrons and s-pelectrons which are present in broad bands. The theory was introduced as the "Electron Interplay Model"16. While this model is relatively crude, ignoring geometrical effects, it allows the explanation of a series of basic catalytic properties, e.g., why $\mathrm{H}_{2}$ dissociates on $\mathrm{Pt}$ but not on $\mathrm{Na}$ while both $\mathrm{Pt}$ and $\mathrm{Na}$ form stable hydrides ( $\mathrm{Pt}$ is a good hydrogenation catalyst). It was attractive to use actinides to test this model because some actinides have highly correlated f-electrons $(\mathrm{Np}$, $\mathrm{Pu}, \mathrm{U}$ ) while others do not ( $\mathrm{Th}, \mathrm{Am}$ ), and comparison of their catalytic properties could tell us something about the role of the highly correlated electrons. This was our starting point in the mid-80s when the authors worked on these projects in Karlsruhe. Unfortunately, it became clear that despite the similarities between actinide $5 f$-electrons and late TM d-electrons, the chemical properties of the elements are very different! The chemical properties of metallic actinides are dominated by the highly reactive $6 \mathrm{~d} 7 \mathrm{~s}$ electrons: actinides do not behave like late but like early TMs owing to their descendence from actinium, which is a refractory metal. We found that the high chemical reactivity of $\mathrm{U}$ was manifested by the exclusive dissociative adsorption of $\mathrm{CO}$ on $U$ metal, even at $-200^{\circ} \mathrm{C}^{14}$. However, we also found that $U$ loses part of its reactivity when diluted in an alloy such as UNi5. This indicated the direction our research had to take: the only way to study the weak bonding properties of the $5 \mathrm{f}$ electrons without interference of the more reactive $6 \mathrm{~d} 7 \mathrm{~s}$ was to have the latter reacted in advance. In other words, we had to look for partially reacted $U$ compounds with $6 \mathrm{~d} 7 \mathrm{~s}$ electrons in stable chemical bonds but with the $5 \mathrm{f}$ electrons still available for bonding. We also discussed another solution: in order to keep $U$ metallic in spite of its high reactivity one could bind it in an alloy with an even more reactive element, e.g., Th, which then would play the role of a buffer and constantly abstract $\mathrm{O}$ and $\mathrm{C}$ atoms from the U. T. Gouder disagreed with this idea because he thought the issue was not to keep $U$ metallic but to keep it from reacting. A catalyst, by definition, is a material which only weakly interacts with the reactants and releases them spontaneously after reaction. One could not achieve this for $U$ by keeping it reactive and adding an even more reactive species to the surface. Let us return to the idea of deactivation of the surface of $U$. There are two ways of achieving this: a) We could partially oxidize the surface forming, e.g., UO, which in contrast to $\mathrm{UO}_{2}$ is still metallic and has $5 \mathrm{f}$ electrons at the Fermi-level. UO is stable in the presence of $\mathrm{C}, \mathrm{S}$, $\mathrm{N}$ as a solid solution of $\mathrm{UO}$ and UC, US, and UN. Thus our interest in the oxycarbide and the 
experiment described above to determine whether surface $\mathrm{UO}$ (or $\mathrm{UO}_{\mathrm{x}} \mathrm{C}_{1-\mathrm{x}}$ ) is stable compared to $\mathrm{UO}_{2}$. b) We could further dilute $\mathrm{U}$ in a substrate, hoping that the $6 \mathrm{~d} 7 \mathrm{~s}$ electrons would become involved in solid-state bonding, enough to make them lose their affinity for chemisorbed molecules, while the $5 f$ electrons, which are more localized, would stay on the $U$ atoms and remain available for bonding. At first sight this seems to be pure speculation, but there are indications that it might be justified. In catalysis, conventional promoters belong to the alkaligroup and there is no question that they are oxidized during the catalytic process. The are usually brought onto the surface in the form of oxides. However, their promoting action is explained, at least by some theories, by electronic interaction with the substrate thereby changing its chemisorption properties. In principle we can expect a similar promoting effect for actinide elements with the $6 \mathrm{~d} 7 \mathrm{~s}$ states involved in bonding with the substrate which simultaneously would deactivate these electrons. In contrast to the alkaline metals, the actinides would have the $5 \mathrm{f}$ electrons available for localized bonding and might behave as local reaction centers (there are analogies with homogenous catalysis where actinide coordination complexes have catalytic properties).

$\mathrm{U}$ deposition provided the opportunity to prepare highly diluted $\mathrm{U}$ surface compounds, which we could use to model U-doped catalysts. Our first experiments had to address the question of whether we could really deactivate the actinide atoms by diluting them in a TM matrix. In this context we studied $\mathrm{CO}$ adsorption on $\mathrm{U}$ overlayers on TM substrates. $\mathrm{CO}$ is often used as a test molecule to determine the reactivity of surfaces. It does not adsorb on inert materials, adsorbs molecularly on low reactive surfaces, and spontaneously dissociates on highly reactive substrates. This leads to the "Volcano Curve" in catalysis, where catalytic activity is suppressed either by too low surface reactivity (no chemisorption) or too high reactivity (reaction of the catalyst with the adsorbate). As mentioned above we found $\mathrm{CO}$ to adsorb dissociatively on pure $\mathrm{U}$ even at $-200^{\circ} \mathrm{C}$ showing it to be far too reactive to behave as a catalyst. We obtained our first results during our study of the U-Pd system. As we discussed earlier, the electronic structure of the Pd surface is dramatically changed even by small amounts of $U$ (the Pd4d emission at the Fermi-level is strongly suppressed [Figure 22]), which is the necessary condition for a promotion (or poison) effect. We then exposed these surfaces to $\mathrm{CO}$ at room temperature (Figure 23). $\mathrm{CO}$ chemisorbed molecularly on pure Pd metal, as seen by the $4 \sigma$ and $5 \sigma / 1 \pi$ molecular orbital emissions; there is no dissociation of $\mathrm{CO}$ as indicated by the absence of the $\mathrm{O} 2 \mathrm{p}$ level (between $5-6 \mathrm{eV}$ ), which is found for atomic $O$ or a surface oxide. With increasing $U$ dosage, the signal of chemisorbed $\mathrm{CO}$ decreases, but only for the highest $\mathrm{U}$ concentration does the signal of atomic oxygen appear. For lower $U$ concentrations $(0.2,0.3)$ there is no indication for $\mathrm{CO}$ dissociation: 


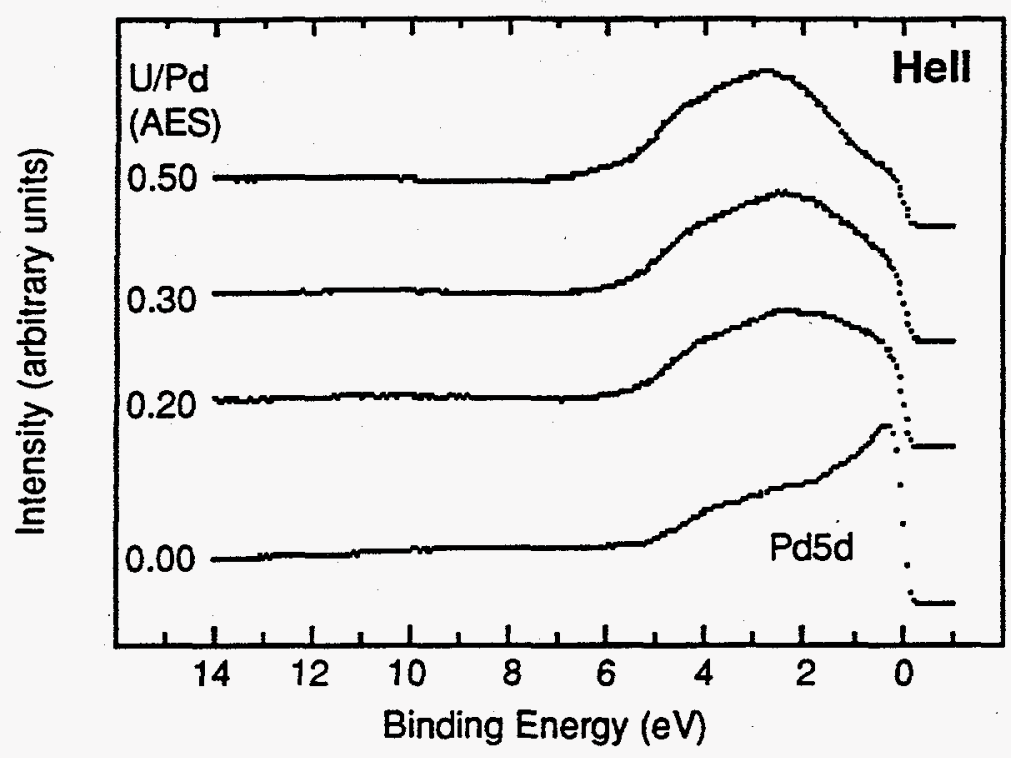

Fig. 22 Hell spectra of U-Pd show that even small amount of $U$ change the electronic structure of $P d$. The (Pd4d) intensity at $E_{F}$ is strongly suppressed.

we made the surface more inert (there is less molecular and no dissociative $\mathrm{CO}$ adsorption) by covering it with small amounts of initially highly reactive $U$ atoms.

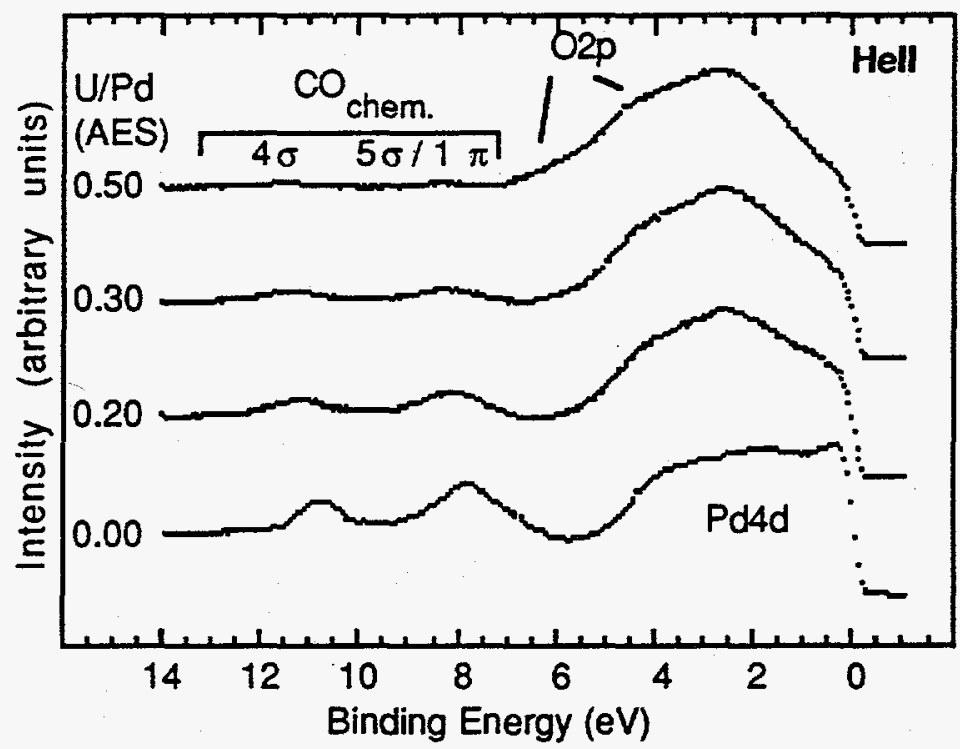

Fig. 23 Hell-study of $\mathrm{CO}$ adsorption on U-Pd at room temperature.

The decrease of surface reactivity at low U coverage can be attributed a priori to two effects: either the surface $\mathrm{Pd}$ becomes covered by $\mathrm{U}$ atoms leaving less $\mathrm{Pd}$ adsorption sites available for $\mathrm{CO}$ molecules, or the chemisorption properties of Pd are changed by $\mathrm{U}$ (e.g. by filling of the 
Pd4d band) resulting in a decrease of the chemisorption energy; the latter would be a purely electronic effect. We tested these hypotheses by following the evolution of the signal of chemisorbed $\mathrm{CO}(\mathrm{CO} 4 \sigma+5 \sigma+1 \pi)$ while heating the surface on which we had previously adsorbed $2 \mathrm{~L} \mathrm{CO}$ at $-156^{\circ} \mathrm{C}$ (Figure 24). Heating resulted in the sudden decrease of the $\mathrm{CO}$ signal (breaking of substrate $\mathrm{C}-\mathrm{O}$ bonds), which can be due either to $\mathrm{CO}$ desorption ( $\rightarrow$ Substrate $+\mathrm{CO}_{\text {gas }}$ ) or $\mathrm{CO}$ decomposition ( $\rightarrow$ Substrate- $\mathrm{C}+$ Substrate-O). Figure 24 shows that the reaction temperature decreased with increasing $U$ concentration. Before discussing this let us first decide whether the reaction is $\mathrm{CO}$ desorption or decomposition. If the decreased reaction temperature was due to $\mathrm{CO}$ reaction with $\mathrm{U}$ then adsorbing $15 \mathrm{~L}$ CO above the reaction temperature should allow completion of this reaction and the transition temperature should sharply increase.

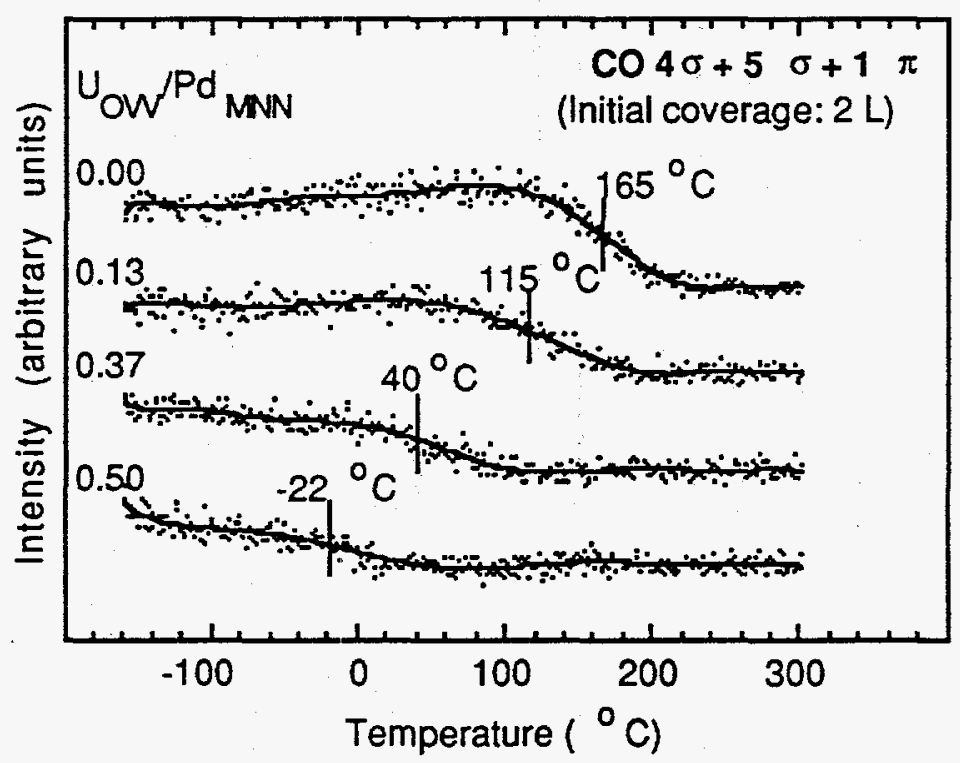

Fig. 24 Evolution of the $\mathrm{CO}_{\text {chem }}$ Auger signal intensity with annealing. With increasing $U$ concentration the reaction temperature decreases. The reaction causes $\mathrm{CO}$ desorption.

If, on the other hand, the reaction is the desorption of $\mathrm{CO}$, then adsorbing $\mathrm{CO}$ above the transition temperature should have no effect: CO simply does not adsorb. The experiment (Figure 25) strongly points to this second explanation: the transition temperature for the U-Pd surface is only slightly affected by $\mathrm{CO}$ adsorption at high temperatures (the small shift to higher temperature is very probably due to the slow decrease of the $U$ surface concentration because of $\mathrm{U}$ bulk diffusion, which already occurs at these temperature [see next section]). From these experiments we conclude that $U$ loses most of its own reactivity and changes the chemisorption properties ( $\mathrm{CO}$ chemisorption energy) of the $\mathrm{Pd}$ substrate. Therefore, the decrease of the $\mathrm{CO}$ 
signal in Figure 23 is at least partially due to the lowering of the chemisorption energy of $\mathrm{CO}$ on Pd $(U$ behaves more like a poison than a promoter, but we expect this to change when choosing other substrates and gases). Supplementary evidence for the weakening of the $\mathrm{Pd}-\mathrm{CO}$ bonding is provided by the $\mathrm{BE}$ difference between the $\mathrm{CO} 4 \sigma$ and $5 \sigma$ emissions $\left(\triangle \mathrm{B}_{\mathrm{E}} 4 \sigma-5 \sigma\right)$ (Figure 26). The increase in $\mathrm{BE}$ between the non-bonding $4 \sigma$ and the $\mathrm{CO}$-substrate bonding $5 \sigma$ by about 0.2 $\mathrm{eV}$ indicates that the $5 \sigma$ orbital energy increases and therefore suggests a weakening of the $\mathrm{CO}$ surface bond ${ }^{17}$. However, we must point out that this argument alone is not very convincing because a) the $\triangle B E$ increase is fairly small (a large $\triangle B E$ increase is not expected since $0.2 \mathrm{eV}$ corresponds to a decrease in chemisorption energy by about $5 \mathrm{kcal} / \mathrm{mole}$, which is already significant), b) there is some arbitrariness in the determination of the maximum because of the $1 \pi$ signal is superimposed on the $5 \sigma$, and c) the correlation between $\triangle B E 4 \sigma-5 \sigma$ and chemisorption energy is far from general ${ }^{17}$. Nevertheless it is interesting to notice that the decreased chemisorption energy in this case seems to be accompanied by the expected increase of $\triangle \mathrm{BE} 4 \sigma-$ $5 \sigma$ and chemisorption energy in this case seems to be accompanied by the expected increase of $\triangle B E 4 \sigma-5 \sigma$. HREELS experiments on single crystal Pd are planned to confirm this.

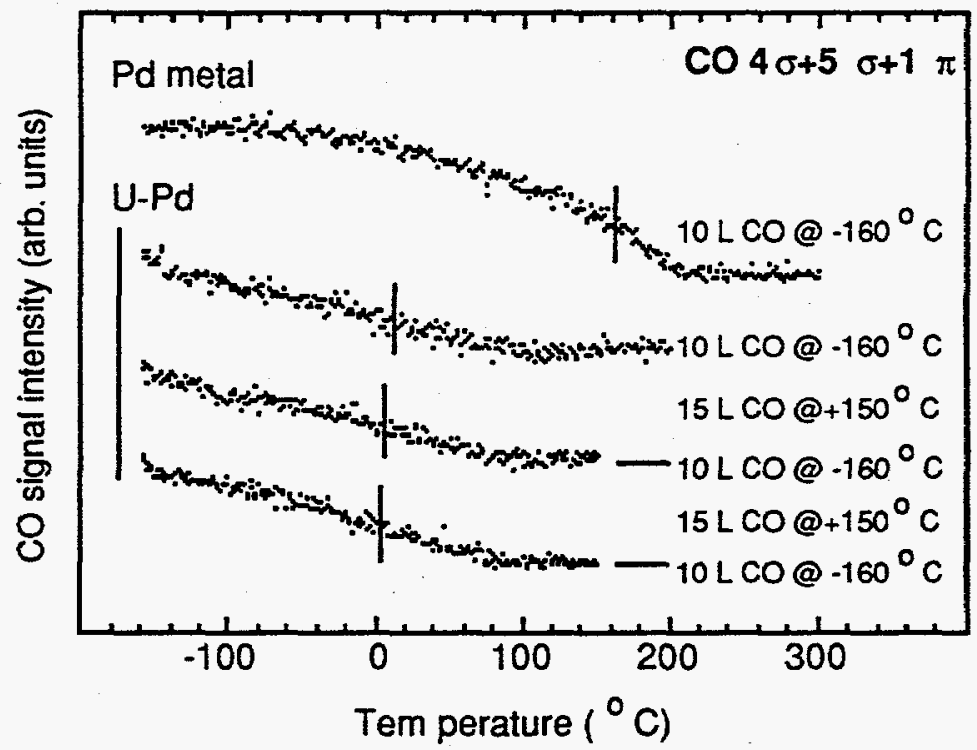

Fig. 25 Evolution of the $\mathrm{CO}_{\text {chem. }}$ signal intensity with annealing. Even repeated exposure of the surface to $\mathrm{CO}$ at high temperature (above the reaction temperature) only little affects the reaction temperature (all five exposures-desorptions have been done on one sample).

The results obtained thus far are encouraging for further studies of actinides as model catalysts. We have suppressed the high initial reactivity of actinide atoms which was the primary obstacle to these studies. The UHV investigations should now be accompanied by some well selected catalysis experiments on model systems with low actinide surface concentrations. 


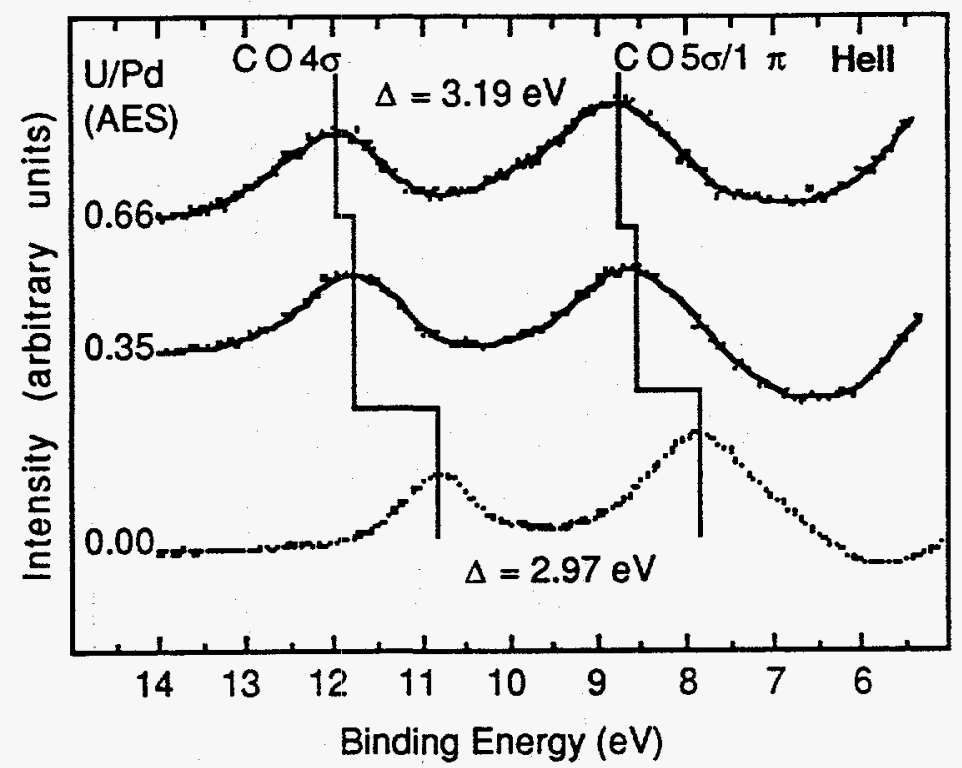

Fig. 26 High resolution UPS spectra of the $\mathrm{CO}_{\text {chem }}$ lines for $\mathrm{CO}$ adsorbed on U-Pd. The $4 \sigma-5 \sigma$ splitting increases with the $U$ concentration. 


\section{Bulk Diffusion of U Overlayers}

Stability of actinide/containment-material interfaces is an important issue in the storage of nuclear waste where thermal decomposition, corrosion, and bulk diffusion of actinide compounds and fission products may present potential problems. Thin layers of actinides on TM substrates offer an opportunity to study these processes. Because of the small amount of initial material even slow processes, which are of importance in long term storage, may be addressed under realistic conditions. Characterization of the overall diffusion reaction is generally complicated by the fact that the actinide-substrate interface constitutes a heterogeneous system and concentration/phase dependent diffusion mechanisms may result in the simultaneous or sequential occurrence of different diffusion-reaction processes. (In the rest of this section we will call "diffusion" any process contributing to the penetration of actinide surface atoms into the substrate; this includes the classical bulk diffusion of highly diluted $U$ atoms and also near surface migration of $U$ during alloying reactions.) Traditional techniques, which study the system after reaction by analyzing the diffusion profile, obtain an integrated result and present difficulties deconvoluting several diffusion processes. Therefore, we thought of a complementary technique which would allow us to differentiate several diffusion mechanisms by their activation energies even if they occur simultaneously. In this section we will briefly describe our approach and present its application in the study of bulk diffusion of $U$ and $U$ compounds into Pd. The major difficulty in our approach is that diffusion parameters in thin layers often differ from bulk diffusion parameters ${ }^{18}$. We will address this briefly at the end of the section.

In a diffusion reaction, the concentration of the diffusing species (A) varies with time and depth: $\mathrm{c}_{\mathrm{A}}=\mathrm{c}_{\mathrm{A}}(\mathrm{x}, \mathrm{t})$. In the classical diffusion experiment, the diffusion is stopped after a fixed time $(\mathrm{t}=$ fixed) and a diffusion profile $c_{A}(x)$ is determined by a sectioning technique (e.g. sputter depth profiling with Auger spectroscopy) or by an analytical technique with depth resolution (Rutherford Back-scattering Spectroscopy, RBS). The diffusion parameters (activation energy, etc.) are determined by carrying out the experiment at different temperatures. In this approach the integrated result of diffusion is measured, and if several diffusion processes are active during the measurement time, either simultaneously (e.g. short circuit diffusion and interstitial diffusion) or consecutively (near surface alloy formation and its subsequent dilution), the technique yields results difficult to deconvolute. In our approach we measure the surface concentration $(x=0)$ of the sample as a function of time $c_{A}(t)$, by following the respective AES signals of adsorbates and substrate (AES is a fast real time technique). Hence, we do not study the system after but during the diffusion reaction. A simple experimental trick allows us to distinguish diffusion processes which differ in activation energy: we ramp the sample temperature during the 
diffusion experiment. (The technique bears strong similarities to thermal desorption spectroscopy (TDS) where a sample surface is also heated linearly and the rate of desorption is measured as a function of temperature and heating rate). At low temperatures diffusion processes with low activation energies predominate. If these reactions do not consume all the diffusing materials, processes with high activation energies will be detected at higher temperatures. We can expect this in two cases: a) the low temperature diffusion is due to grain boundary diffusion which has only limited diffusion channels and tends to be saturated (i.e., temperature increase does not produce an increase in diffusion rate) and b) the low temperature diffusion is coupled to a chemical reaction, e.g. near surface alloy formation, where the reaction enthalpy provides the driving force and the diffusion stops after $A$ is transformed into Ay $(S=$ substrate) and is still present in detectable concentrations within the information depth of AES. A typical diffusion experiment is shown in Figure 27. A Pd sample is covered by a U overlayer and heated linearly to $900^{\circ} \mathrm{C}$. We followed the evolution of the $U$ and $\mathrm{Pd}$ Auger intensities $\left(\mathrm{V}_{\mathrm{p}-\mathrm{p}}\right.$ or integrated peak areas) with temperature and time. To have more direct access to the rate of diffusion we construct the differentiated curved (dI/dt), where the peaks correspond to diffusion processes with different activation energies. Notice that, as in TDS, the peaks are produced by the counteracting effects of rate increase due to increasing temperature and rate decrease due to decreasing concentration of the diffusing species: they are a consequence of the ramping of temperature. While the mathematical description of such processes is well established, the finite

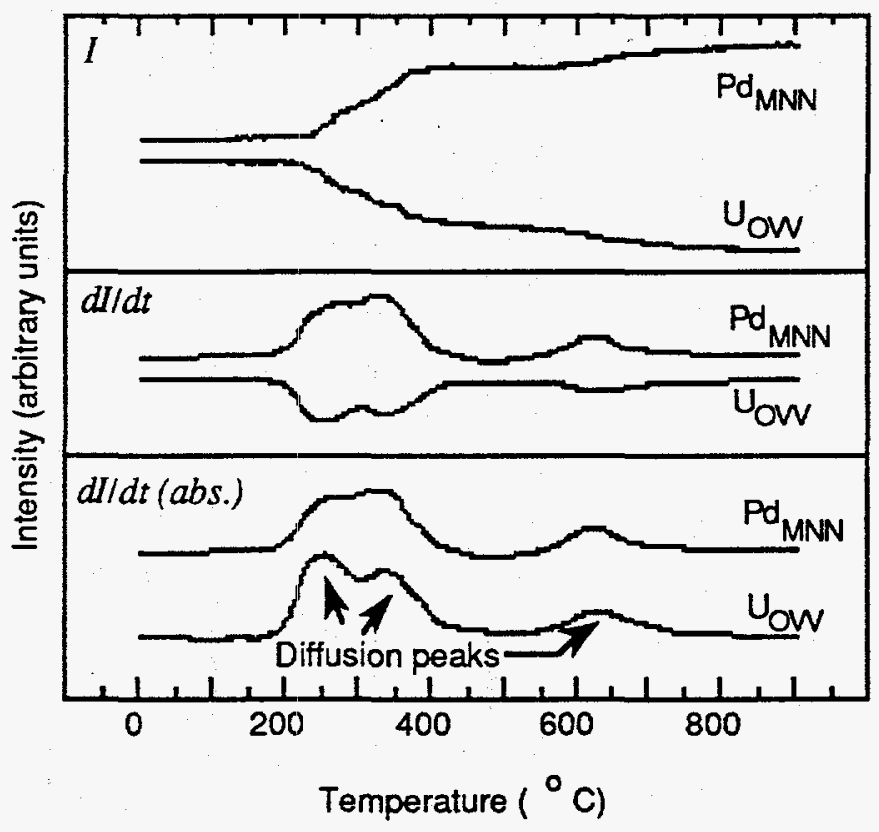

Fig. 27 Diffusion curves: evolution of the $\mathrm{Pd}_{\mathrm{MNN}}$ and $\mathrm{H}_{\mathrm{OW}} \mathrm{AES}$ signal intensities with time. The temperature is raised linearly at a rate of $1^{\circ} \mathrm{C} / \mathrm{sec}$. For discussion of diffusion rates, the initial $\mathrm{l}(\mathrm{t}) \mathrm{curve}$ is differentiated and for easier comparison the absolute values of the derivative are displayed. 
information depth of AES introduces further complications. When these are solved it should be possible to apply the experimental tricks of TDS, such as different heating rates and profiles, to obtain further information on the diffusion process. However, this technique presents a few disadvantages, e.g., the poorly defined information depth which makes it difficult to use AES to determine surface concentrations. Therefore, it should be accompanied by sputter depth profiling which can be performed with the same spectrometer.

We used this technique to study the near surface and bulk diffusion of $\mathrm{U}, \mathrm{UO} \mathrm{O}_{2}$, and $\mathrm{UO}_{\mathrm{x}} \mathrm{C}_{1-\mathrm{x}}$ on a polycrystalline Pd substrate. The polycrystalline surface introduces further complications such as grain boundary diffusion. Therefore, it does not make sense to discuss the results in detail but we will save this for an imminent study on single crystal Pd and limit the discussion here to some representative results and a short intuitive discussion. Figure 28 shows Pd concentration curves for several initial $U$ coverages. For high $U$ concentrations the initial Pd signal is weak because the sample is covered by a thick $U$ reaction layer (U-Pd alloy, $U$ islands). Notice, however, that the Pd signal does not vanish. All curves show regions of constant slope and s-shaped (sigmoidal) regions. The latter are associated with the onset of a diffusion process at a given temperature. Thus, it is clearly seen that there are several diffusion processes for $\mathrm{U}$ diffusion into

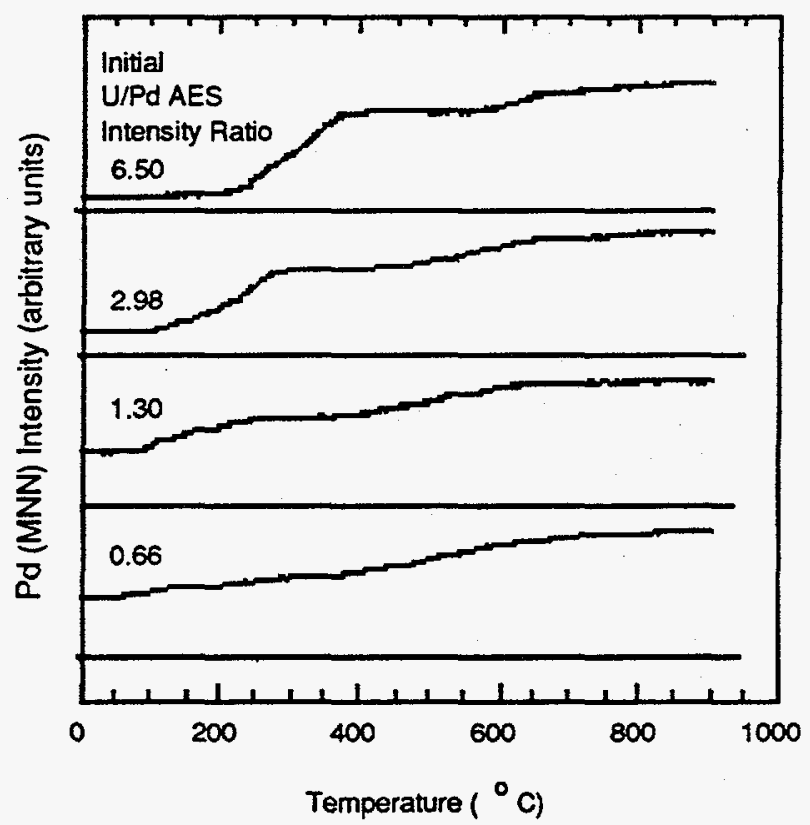

Fig. 28 Annealing curve (Pd signal) for different initial $U$ coverages. The curves show regions of sudden slope increase which correspond to onsetting diffusion processes.

Pd. In areas of constant slope either no diffusion is detected (zero slope) or, at least, no new diffusion process is active (non zero slope). 
Let us discuss these regions in some more detail. The lowest linear region starts at room temperature. Its upper limit moves to higher temperatures with higher $U$ dosage. At very low dosage the slope is positive even at the lowest temperatures showing diffusion processes to be active below $100^{\circ} \mathrm{C}$ (the AES electrons may be involved in the activation of room temperature diffusion). With higher $U$ dosages the slope becomes zero even though the low temperature diffusion processes are active, as shown by the low coverage data. The fact that the $U$ diffusion into Pd is no longer seen in AES shows that it occurs beyond the AES information depth: the reaction takes place at the base of thick islands from where Auger electrons can no longer escape (Figure 29).

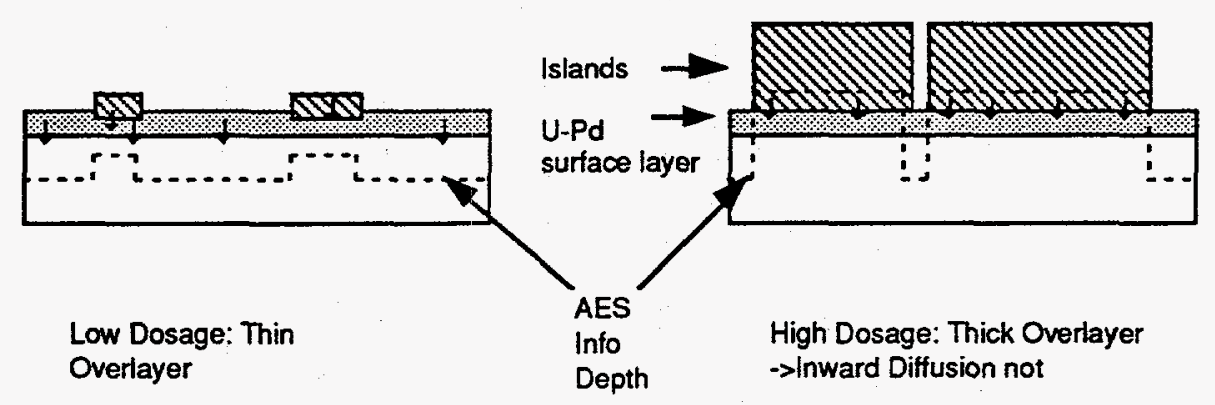

Fig. 29 Island dissolution: For thick islands the dissolution does not change the AES signal, because AES does not look through the layer

In spite of the thick $U$ layers, a non zero Pd signal is detected at the beginning. This shows that the surface is not covered uniformly by a $U$ overlayer but by thick islands which grow on the $U-$ $\mathrm{Pd}$ reaction layer. The slope of the flat region at medium temperature is also a function of initial surface coverage (Figure 28): at low coverage it is positive while at high coverage it is zero. Our interpretation is the following. The low temperature reaction leads to the formation of a thick UPd 3 near-surface alloy (Figure 30 ) which then dissolves at higher temperatures. The dissolution starts at the bottom and is only detected by AES when it reaches the top (Figure 31). This results in the zero slope. Let us discuss the experimental evidence. Formation of UPd $\mathrm{UP}_{3}$ at low temperature was already indicated by the evolution of the electronic structure (localization of the 5 f electrons, Figure 8). In addition, a Pd signal of about $75 \%$ of its final value in pure $\mathrm{Pd}$ (Figure 28) suggests the formation of a Pd compound of 75 at \% Pd, I.e., UPd3. This U-Pd alloy layer can be expected to be about four times thicker than the initial $U$ overlayer (Figure 30). 
U

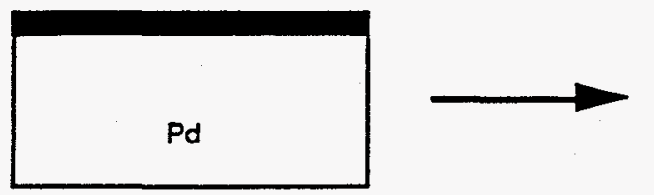

Pd covered by $U$ overlayer
UPd 3

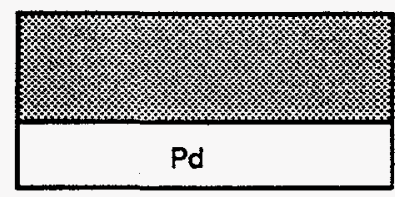

Near surface alloy formed by interdiffusion is about four times thicker than the initial overlayer

Fig. 30 Diffusion of a $U$ layer produces an interdiffusion layer UPd 3 of about four times the thickness of the initial overlayer. Partial interdiffusion takes places immediately after deposition, so the initial overlayer drawn is only hypothetical.

$\mathrm{UPd}_{3}$ will react with the Pd substrate to form more diluted U-Pd alloys (UPd4, $\mathrm{UPd}_{5}, \mathrm{U}_{2} \mathrm{Pd}_{11}$, and $\mathrm{U}_{2} \mathrm{Pd}_{17}$ are stable as bulk phases) and finally dissolve in the Pd substrate ${ }^{19}$. The reaction will start at the interface between $\mathrm{UPd}_{3}$ and the more highly diluted phase and proceed to the surface. For otherwise identical conditions (same heating rate) this will take longer the thicker the UPd 3 near-surface alloy. This explains the apparent delay (or high temperature shift) of the high temperature diffusion process with increasing $U$ dosage. Thus, the exact position of the diffusion peaks directly depends on the amount of $U$ initially deposited. The correct determination of the initial coverage is therefore crucial for a quantitative evaluation of the data.

Figure 32 shows a more extensive set of $\mathrm{Pd}_{\mathrm{MNN}}$ diffusion curves for increasing $\mathrm{U}$ coverage. The differentiated curves show a low and a high temperature peak system. The low temperature system (peaks A, B, and C) is weak at low U dosages but becomes dominant at higher dosages. This is attributed to the reaction of the $U$ accumulation layer ( $U$ overlayer and islands) with Pd. At low coverage there is no $\mathrm{U}$ accumulation and, therefore, no low temperature reaction, but with increasing dosages $U$ accumulates at the surface and the instability of the system triggers $U$
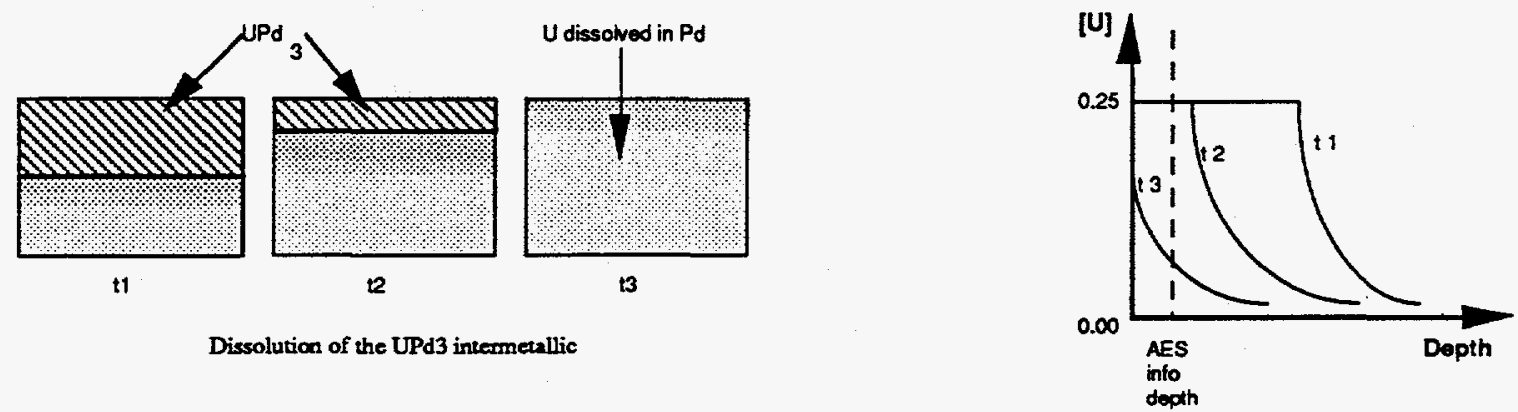

Fig. 31 Advance of the reaction front to the surface $(t 1<t 2<t 3)$

diffusion into the near surface region. The low temperature system consists of two or three superimposed peaks (A, B, and C); the lowest temperature peak (A) is more prominent at low 
coverage and then saturates, while the highest temperature peak (B) appears only at higher coverage. It is tempting to assign them to near surface diffusion of the $U$ overlayer and $U$ islands because the overlayer forms first and the islands grow on in later. However, one would have to conclude that the overlayer dissolves before the islands do, which is not evident. At high coverages $(5.30,6.50)$ either peak A disappears and a new diffusion peak $(C)$ appears or peak $A$ suddenly moves to higher temperatures where it appears as peak $\mathrm{C}$. We will leave a more extensive discussion to the single crystal study because of the possible influence of grain boundary processes on different crystal planes. The high temperature region shows one diffusion peak (D), which is broad at low coverage but narrows and shifts to higher temperature with increasing coverage. The high temperature shift is due to the extension of the diffusion region (Figure 31). The narrowing is due to the increased temperature: once the reaction front reaches the surface the drop in $U$ concentration is faster at higher temperature.

Let us now discuss the bulk diffusion of $\mathrm{U}$ oxycarbide and $\mathrm{UO}_{2}$ in $\mathrm{Pd}$ and compare it to $\mathrm{U}$ metal diffusion. We synthesized a thin layer of $U$ oxycarbide on Pd by exposing the $U$ overlayer to a saturation dosage of $\mathrm{CO}(30 \mathrm{~L})$. Figure 33 shows the changes of the diffusion peaks as a function of the initial $U$ coverage. When comparing it to Figure 32 we first notice that the low temperature peaks of Figure 32 either completely disappear (Peak A) or appear only at higher U dosages (peak 32B could correspond to peak 33A that, however, is shifted to higher

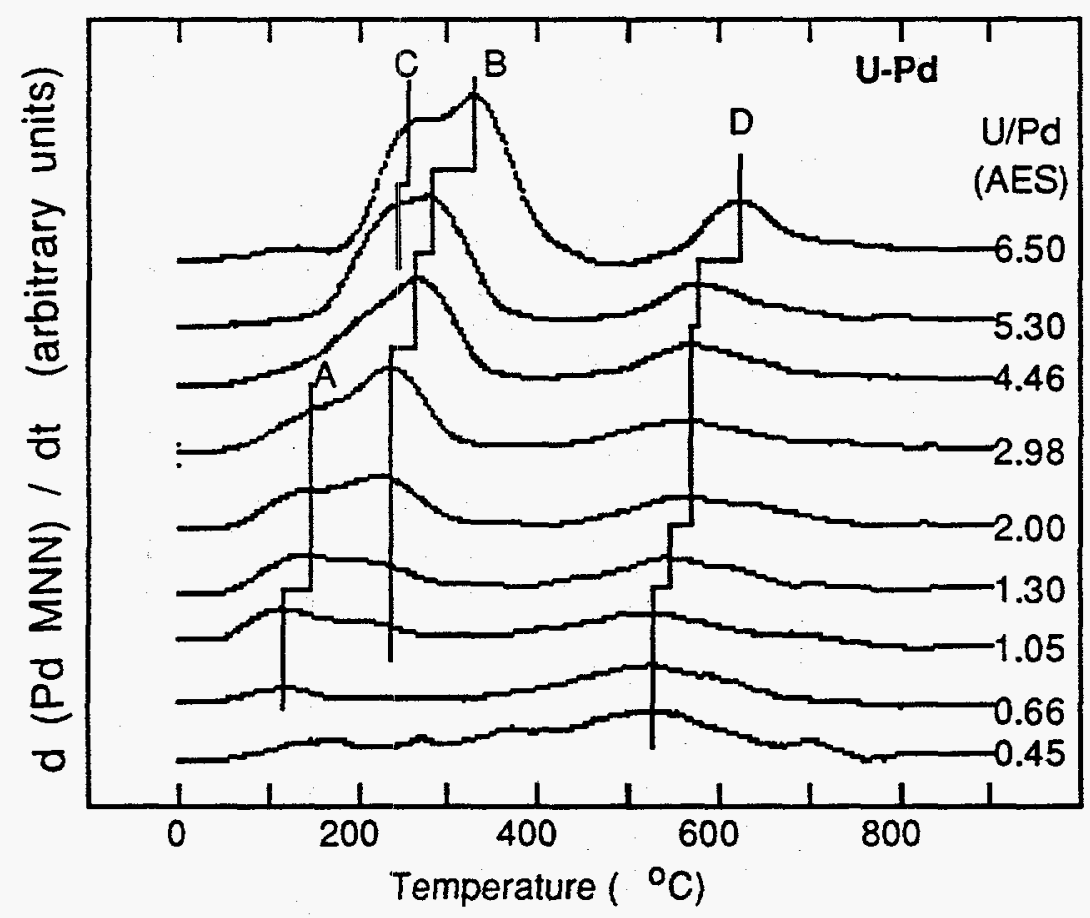

Fig. 32 Diffusion curves for U-Pd as function of the initial $U$ coverage. 
temperatures). At low $\mathrm{U}$ coverage $\mathrm{CO}$ reacts with the uranium responsible for the low temperature peaks $32 \mathrm{~A}$ and $32 \mathrm{~B}$ (the $\mathrm{U}$ coverage is above the threshold coverage of 0.5 where $\mathrm{CO}$ starts dissociating. See previous section). Because we know from a previous study that $\mathrm{CO}$ reacts only with surface $U^{14}$, we conclude that at low coverages $U$ is located on the surface . (This was already indicated by the low inelastic background in Figure 4). This again indicates that peak $32 \mathrm{~A}$ is due to a $\mathrm{U}$ overlayer. Peak $32 \mathrm{~B} / 33 \mathrm{~A}$ could be attributed to island dissolution: at low $U$ coverage, the islands are thin and all island $U$ reacts with $C O$, therefore, peak $33 \mathrm{~A}$ is suppressed at dosages where 32B is already present, while at higher coverages the islands become thicker and contain $U$ atoms that no longer are accessible to $\mathrm{CO}$ and produce the island dissolution peak 33A. Peak 33C corresponds to peak 32D and is attributed to the high temperature dissolution of $\mathrm{UPd}_{3}$. It grows with $\mathrm{U}$ coverage when $\mathrm{U}$ deposits in thick islands and does not react with $\mathrm{CO}$ but with $\mathrm{Pd}$ (peak 33A) to form UPd3. However, it is also seen at low coverage where apparently some of the surface $U$ does not react with CO but stays in the U-Pd intermetallic form (this has to be expected because of the decreased reactivity of $U$ ). Peak 33D corresponds to the oxide decomposition peak: it coincides with the $\mathrm{O}_{\mathrm{KLL}}$ diffusion peak (Figure 34). At low coverage, where the $U$ concentration is still high enough to produce $C O$ dissociation, it is already large but seems to saturate at high coverage, which is in agreement with the formation of a mono-atomic oxide layer. Peak 33B is as yet unexplained. It might be due to the decomposition of $U$ carbide (we cannot follow the $C_{K L L}$ AES peak because it overlaps with intense $\mathrm{U}$ and Pd Auger lines).

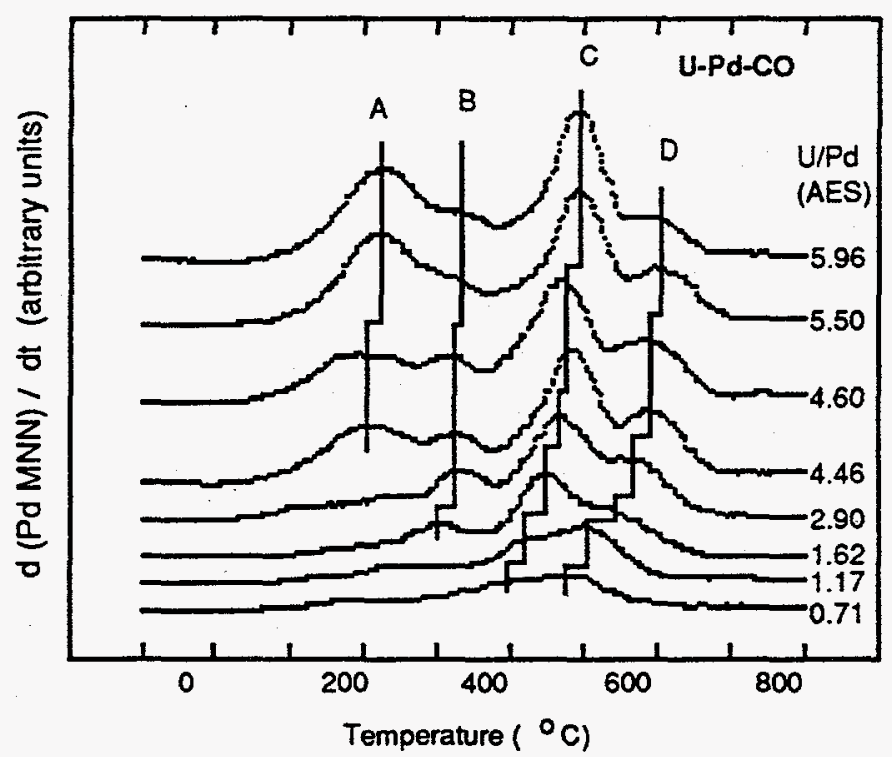

Fig. 33 Diffusion curves for U-Pd-CO as function of the initial U coverage. A saturation dosage of CO produced an oxycarbide overlayer. Disappearance of the low temperature diffusion peaks of Fig. 32 shows the latter to be due to surface $U$ which in this experiment reacted with $C O$. 
Figure 35 shows the diffusion of a $\mathrm{U}$ overlayer that we exposed to $5 \mathrm{~L} \mathrm{O}_{2}$. All three curves have only one diffusion peak suggesting a single diffusion process which is coupled to oxide decomposition ( $\mathrm{O}_{\mathrm{KLL}}$ peak). All other diffusion processes (low temperature alloy formation and $\mathrm{UPd}_{3}$ decomposition) have disappeared showing the surface to be covered by $\mathrm{UO}_{2}$. These results are in agreement with the UPS-study (Figure 14) and XPS-study (Figure 16) of $\mathrm{O}_{2}$ adsorption on U-Pd, both of which show $\mathrm{O}_{2}$ adsorption to result in the decomposition of the $\mathrm{UPd}_{3}$ interdiffusion alloy and in the segregation of $U$ to the surface where it reacts with $O$ to form $\mathrm{UO}_{2}$.

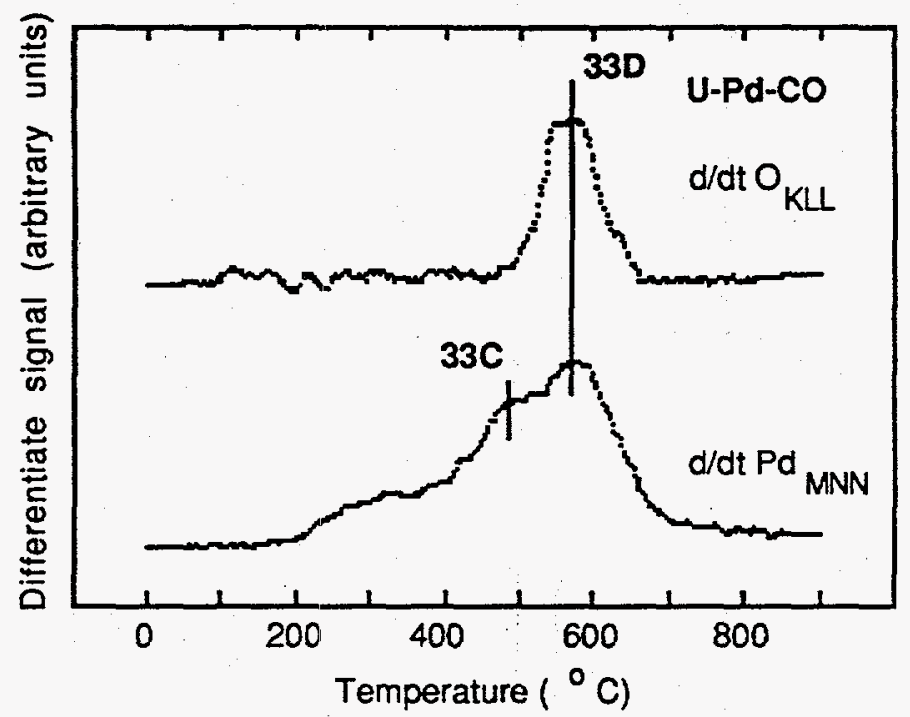

Fig. $34 \mathrm{O}$ and $\mathrm{Pd}$ diffusion curves for UPd=0.71 for $\mathrm{CO}$ adsorption. Peak $33 \mathrm{D}$ is due to oxide decomposition. (At higher $U$ dosages other weak $O_{K L L}$ diffusion peaks develop at lower temperatures but peak 33D always stays dominant.)

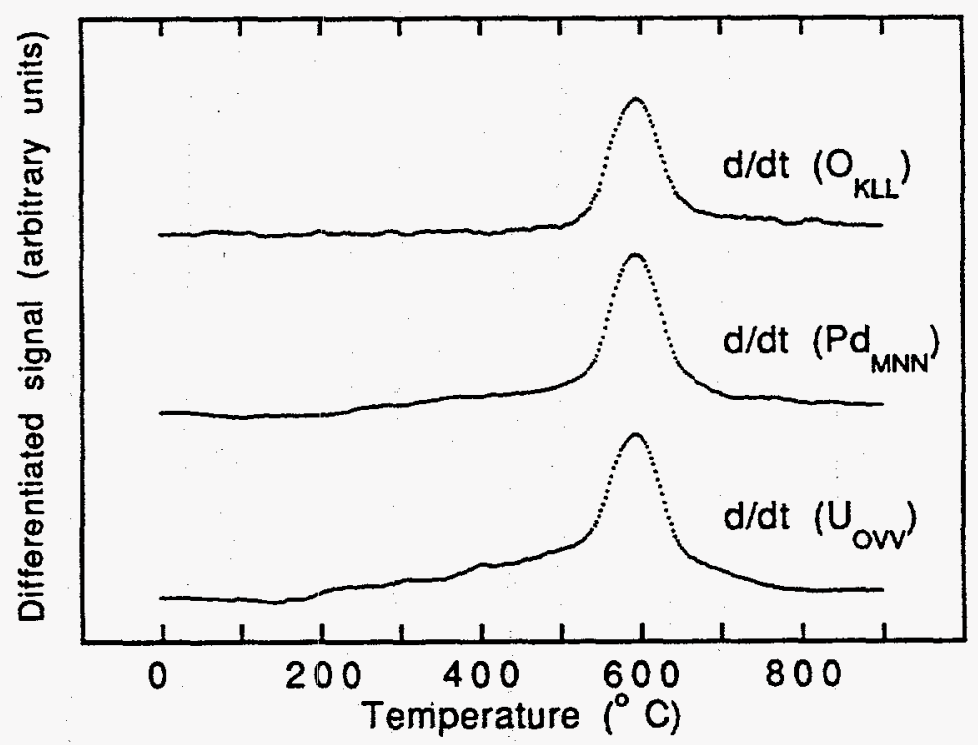

Fig. $35 \mathrm{O}, \mathrm{Pd}$ and $U$ diffusion curves for $U / \mathrm{Pd}=0.71$ after $\mathrm{O}_{2}$ adsorption. The coincidence of all three curves shows only one diffusion process to be active and to be associated with the decomposition of $\mathrm{UO}_{2}$. 
The above study, even though preliminary, shows our measurements of the diffusion behavior of $U$ to be sensitive to the different chemical forms of $U$ on the surface. In addition, it is capable of distinguishing several diffusion processes that may occur either simultaneously ( $\mathrm{UPd}_{3}$ decomposition/Oxide decomposition) or sequentially ( $\mathrm{UPd}_{3}$ formation/dissolution). Even though significant data modeling, which is more complicated than for TDS, must be done to produce quantitative results, the technique at this stage already allows qualitative statements about the diffusion behavior of thin surface films to be made. There are, however, weak points in our approach. The two major ones are a) that AES does not allow us to determine exactly the composition of the uppermost layer, but it gives a weighted sum over several layers, and b) that because of the minute amount of material and the shallow information depth we record, the very early stages of diffusion, which take place in the surface region, may differ from bulk diffusion (because of surface reconstruction, lattice relaxation, enhanced grain boundary diffusion, etc.). Therefore, the results will not always be representative of bulk diffusion, However, there are many experimental and theoretical studies which permit us to relate surface and bulk kinetics and thermodynamics, and there are experimental remedies which should allow us to get bulk sensitive diffusion data even with AES (e.g., the surface accumulation method ${ }^{20}$ ). If we can overcome these problems our approach may be complementary to the more classical diffusion studies. 


\section{Conclusions}

Study of thin layers contributes to the scientific understanding of actinide systems. It allows investigation of issues which are closely related to the handling and long term storage of nuclear materials and waste. Some prominent features of our approach are:

- We can prepare non-buried actinide-substrate interfaces and use well established surface analysis tools to study their corrosion resistance, decomposition under heat, and, on a more fundamental level, their electronic structure and chemical reactivity.

- We can prepare an actinide phase consisting of minute amounts of material so that even very slow chemical reactions (e.g., diffusion at low temperatures) will yield measurable results.

- We can prepare actinide systems with variable stoichiometry and even with compositions which are not stable in the bulk. This should enable us to investigate chemical properties (reaction temperature, chemisorption properties...) as a function of the actinide concentration.

- Thin layers accentuate the narrow band properties of actinides because of the decreased coordination. Applications lie in the fields of magnetism (enhanced surface magnetism), superconductivity, heavy fermion systems, and catalysis.

- The decreased reactivity of $U$ at low concentrations make it possible to investigate its potentially useful catalytic properties. 


\section{Authors' Comments}

Dr. Gouder was happy to initiate this research at Livermore with Dr. Carlos Colmenares. It is a scientific continuation of the $\mathrm{PhD}$ thesis work he did with Dr. Jochen Naegele at Karlsruhe. We think that the intense thin layer research in Lanthanides should have its counterpart in Actinides, which show even more versatile chemical and physical properties that are very sensitive to the particular environment in thin layers. As we have pointed out, thin layers may offer an opportunity to investigate long term storage of nuclear waste. Research in this field could range from the empirical evaluation of containment materials and the study of the influence of radiation-induced defects on bulk diffusion to a more fundamental study of the slow and early diffusion processes themselves. Part of our data are preliminary, but we believe they show the potential value of this research. 


\section{References}

1. J.E. Inglesfield, Re. Prog. Phys. 45 (1982), 223.

2. A.J. Freeman, Physica 102 B (1980), 3.

3. R.J. Tench, M. Balooch, W.J. Siekhaus, W. McLean II, STM Conference (Lausanne), 1990.

4. T. Gouder, C.A. Colmenares, Surface Science 295 (1993), 241.

5. S. Doniach and M. Sunjic, J. Phys. C 3 (1970), 284.

6. R. Baptist, D. Courteix, J. Chayrouse, and L. Heintz, J. Phys. F12 (1982), 2103.

7. J.R. Naegele, L. Manes, J.C. Spirlet, and W. Müller, Phys. Rev. Lett. 52 (1984), 1834.

8. Y. Baer, H.R. Ott, and K. Andres, Solid State Commun. 36 (1980), 387.

9. W.-D. Schneider and C. Laubschat, Phys. Rev. Lett. 46 (1981), 1023.

10. A.J. Arko, D.D. Koelling, B.D. Dunlap, A.W. Mitchell, C. Capasso, and M. del Giudice, J. Appl. Phys. 62 (1988), 3680.

11. J.N. Andersen, J. Onsgaard, A. Nilsson, B. Eriksson, and N. Martensson, Surface Sci. 202 (1988), 183.

12. R.J. Tench et. al., LLNL, unpublished results (1992).

13. G. Rossi, Surface Sci. Rep 7 (1987), 1.

14. T. Gouder, C.A. Colmenares, J.R. Naegele, J.C. Spirlet, and J. Verbist, Surface Science 264 (1992), 354.

15. T.H. Gouder, C. Colmenares, J.R. Naegele, and J. Verbist, Surface Sci. 235 (1989), 280.

16. Z. Knor, in Catalysis (Specialist Periodical Report), The Royal Society of Chemistry, London (1985), Vol. 7, 148.

17. J. Rogozik and V. Dose, Surface Sci. 176 (1986), L847.

18. R.W. Balluffi and J.M. Blakely, Thin Solid Films 25 (1975), 363.

19. U. Gösele, in Alloying (J.L. Walter, M.R. Jackson, C.T. Sims, eds.), ASM International, Metals Park, Ohio (1988), 489.

20. J.C.M. Hwang, J.D. Pan, and R.W. Balluffi, J. Appl. Phys. 50 (1979), 1349. 